Comment. Math. Helv. 76 (2001) 61-88 $0010-2571 / 01 / 010061-28 \$ 1.50+0.20 / 0$
(C) 2001 Birkhäuser Verlag, Basel

Commentarii Mathematici Helvetici

\title{
Limites d'espaces tangents à une surface normale
}

\author{
Jawad Snoussi
}

\begin{abstract}
Résumé. Nous étudions l'ensemble des hyperplans limites d'hyperplans tangents à un germe de surface normale. Nous caractérisons ces hyperplans par le fait que le nombre de Milnor de leur section avec la surface n'est pas minimum. Nous donnons ensuite une généralisation des résultats de [14] en termes de résolution simultanée faible de la famille des sections hyperplanes, ce qui nous permet de déterminer avec précision les tangentes exceptionnelles d'une surface normale. Grâce à ces résultats, nous démontrons que "les composantes de Tyurina" d'une désingularisation raisonnable se contractent sur des points fixes du système linéaire des courbes polaires.
\end{abstract}

\begin{abstract}
We study the set of limiting tangent hyperplanes of a normal surface germ. We characterize these hyperplanes by the non-minimality of the Milnor number of their section with the surface. Then we generalise the results of [14] in terms of weak simultaneous resolution of the family of hyperplane sections, and hence we precisely determine the exceptional tangents of a normal surface singularity. Applying these results, we prove that the "Tyurina components" of a reasonable desingularisation contract to fixed points of the linear system of polar curves.
\end{abstract}

Mathematics Subject Classification (2000). 32S05, 32S15, 32S25, 32S35, 32S45.

Mots-clès. Surface normale, Limite d'espaces tangents, Tangente exceptionnelle, Nombre de Milnor, Courbe polaire, Composante de Tyurina.

\section{Introduction}

L'étude de la géométrie locale d'un espace analytique complexe $X$ au voisinage d'un point singulier $x$ est intimement liée au comportement des espaces tangents à $X$ au voisinage de $x$. Ce point de vue étudié notamment par H. Whitney, D.T. Là et B. Teissier (cf. [30], [14], [13], [27], [17] ...) a largement contribué à la classification et à l'étude de l'équisingularité des espaces analytiques.

Considérons un espace analytique équidimensionnel $X$ plongé dans $\mathbb{C}^{N}$ de dimension $d$ et $x$ un point singulier de $X$. Notons $\nu$ l'application qui associe à tout point non-singulier de $X$ la direction de l'espace tangent à $X$ en ce point. L'adhérence du graphe de $\nu$ dans $X \times \mathrm{G}(d, n)$ munie de la restriction de la première projection $\mu: \tilde{X} \rightarrow X$ est la modification de Nash de l'espace $X$. La fibre $\mu^{-1}(x)$ est identifiée à l'ensemble des directions limites d'espaces tangents à 
$X$ en $x$.

Cet ensemble n'est connu explicitement que dans certains cas particuliers. Dans le cas des courbes, c'est l'ensemble des composantes irréductibles du cône tangent. Dans le cas des hypersurfaces à singularités isolées de $\mathbb{C}^{3}$, J.P.G. Henry et D.T. Là en donnent une description explicite dans [14]. Une caractérisation numérique dans le cas des hypersurfaces à singularités isolées est donnée par B. Teissier dans [27]. Des caractérisations géométriques sont également faites dans le cas des surfaces dans [13] et dans des situations générales dans [16] et [17].

Dans ce travail, nous nous consacrons au cas des surfaces normales plongées dans $\mathbb{C}^{N}$. Un hyperplan tangent à la surface en un point non-singulier est un hyperplan de $\mathbb{C}^{N}$ qui contient le plan tangent à la surface en ce point. Nous étudions en détail l'ensemble des limites d'hyperplans tangents à une surface normale en des suites de points non-singuliers qui convergent vers ses points singuliers. L'idée de considérer des hyperplans tangents, comme dans [17], permet dans le cas des surfaces normales d'exprimer les résultats plus simplement. Nous remplaçons pour cela la modification de Nash par le morphisme conormal. Plus précisément nous donnons une généralisation des résultats connus dans le cas des hypersurfaces de $\mathbb{C}^{3}$ à singularités isolées.

Dans [27], B. Teissier caractérise les hyperplans qui ne sont pas limites d'hyperplans tangents à une hypersurface à singularités isolées par la minimalité du nombre de Milnor de la section hyperplane aux points singuliers. Nous démontrons (théorème 4.2) que cette caractérisation est encore valable dans le cas des surfaces normales. Pour cela, nous avons besoin d'une définition du nombre de Milnor pour des courbes gauches. Nous utiliserons la généralisation du nombre de Milnor pour une courbe gauche réduite donnée dans [1]. Une telle approche pourrait être généralisée aux dimensions supérieures si l'on dispose d'une définition raisonnable d'un nombre de Milnor généralisé.

Dans [16] (§2.1), D.T. Là et B. Teissier mettent en évidence sur le cône tangent d'une surface $\mathrm{S}$ en un point singulier $\xi$, un nombre fini de génératrices, dites tangentes exceptionnelles de la surface en $\xi$, qui permettent de décrire l'ensemble des limites d'hyperplans tangents à la surface au voisinage de $\xi$ comme réunion de l'ensemble des hyperplans tangents au cône tangent le long de ses génératrices et de l'ensemble des hyperplans contenant une tangente exceptionnelle. Dans [14], J.P.G. Henry et D.T. Là, démontrent que les tangentes exceptionnelles d'une hypersurface de $\mathbb{C}^{3}$ à singularités isolées correspondent aux points, dans les courbes exceptionnelles des éclatements des points singuliers, où il y a perte d'équisingularité de la surface éclatée le long des courbes exceptionnelles. En utilisant la notion de résolution simultanée faible (cf. [26] et [1] $\S \S 4$ et 5 ), nous démontrons, dans le cas des surfaces normales de $\mathbb{C}^{N}$ (théorème 5.6), que les tangentes exceptionnelles correspondent aux points où, localement, la famille des sections hyperplanes n'admet pas de résolution simultanée faible. Ce qui nous permettra de déterminer avec précision les tangentes exceptionnelles d'une surface normale (théorème 5.8). Nous en déduisons une relation entre les sections 
hyperplanes que G. González-Sprinberg et M. Lejeune-Jalabert appellent sections hyperplanes générales dans [9] (définition 4) et les sections hyperplanes définies par des hyperplans qui ne sont pas des limites d'hyperplans tangents à la surface en ses points singuliers.

Dans [21] (III. remarque 3.12), M. Spivakovsky pose la question de savoir si les points singuliers de l'éclatement des singularités d'une surface à singularités rationnelles sont des points fixes du système linéaire des courbes polaires dans l'éclatement. En remarquant que les tangentes exceptionnelles sont les tangentes fixes du système linéaire des courbes polaires, nous pouvons répondre positivement à cette question dans le cas général des surfaces normales (théorème 6.6). Nous donnons également une formulation de ce résultat en terme de contraction des composantes de Tyurina d'une désingularisation de la surface (théorème 6.9). Pour cela, en utilisant un résultat de [9] sur la contraction des composantes irréductibles $\mathrm{du}$ diviseur exceptionnel dans une résolution raisonnable, nous expliquons que la notion de composantes de Tyurina introduite pour les singularités rationnelles de surface dans [29], se prolonge au cas des surfaces normales comme l'a remarqué M. Spivakovsky dans [21] (III. lemmme 7.1).

\section{Définitions et résultats généraux}

Soit $\left(X, x_{0}\right)$ un germe d'espace analytique équidimensionnel réduit de dimension $d$ que l'on supposera plongé dans $\left(\mathbb{C}^{N}, 0\right)$. Nous désignerons par $X$ un représentant "assez petit" du germe $\left(X, x_{0}\right)$ et par $X_{0}$ le lieu non-singulier de $X$.

Notons $\mathcal{O}_{X, x_{0}}$ l'anneau local des fonctions holomorphes définies sur $X$ au voisinage de $x_{0}$.

\subsection{Espace conormal}

Considérons le sous-ensemble $\mathrm{C}_{0}\left(X_{0}, \mathbb{C}^{N}\right)$ de $X_{0} \times \check{\mathbb{C}}^{N}$ constitué des couples $(x, \xi)$ où $x \in X_{0}$ et $\xi: \mathbb{C}^{N} \rightarrow \mathbb{C}$ est une forme linéaire qui s'annule sur l'espace tangent, $\mathrm{T}_{x} X_{0}$, à $X_{0}$ en $x$.

Définition 2.1. L'adhérence dans $X \times \check{\mathbb{P}}^{N-1}$ du projectivisé par rapport au second facteur de l'espace $\mathrm{C}_{0}\left(X_{0}, \mathbb{C}^{N}\right)$ est appelée espace conormal associé à $X$ dans $\mathbb{C}^{N}$; on le note $\mathrm{C}\left(X, \mathbb{C}^{N}\right)$. On note $\chi: \mathrm{C}\left(X, \mathbb{C}^{N}\right) \rightarrow X$ le morphisme induit par la première projection et on l'appelle morphisme conormal.

L'espace conormal $\mathrm{C}\left(X, \mathbb{C}^{N}\right)$ est un sous-espace analytique de $\mathbb{C}^{N} \times \check{\mathbb{P}}^{N-1}$ de dimension $N+d-1$ (cf. [25]).

Si $x$ désigne un point non-singulier de l'espace $X$, l'ensemble $\left|\chi^{-1}(x)\right|$ correspond à l'ensemble des hyperplans de $\mathbb{C}^{N}$ tangents à l'espace $X$ en $x$. 
On dit qu'un hyperplan $H$ de $\mathbb{C}^{N}$ est une limite d'hyperplans tangents à l'espace $X$ en $x_{0}$ s'il existe une suite de points non-singuliers $\left(x_{n}\right)$ de $X$ qui converge vers $x_{0}$ et une suite d'hyperplans $\left(H_{n}\right)$ de $\mathbb{C}^{N}$ telles que pour tout $n$ l'hyperplan $H_{n}$ soit tangent à $X$ en $x_{n}$ et la suite $\left(H_{n}\right)$ converge vers $H$ dans

$\check{\mathbb{P}}^{N-1}$. Le projeté sur $\check{\mathbb{P}}^{N-1}$ de l'ensemble $\left|\chi^{-1}\left(x_{0}\right)\right|$ est l'ensemble des hyperplans limites d'hyperplans tangents à $X$ en $x_{0}$. On l'identifiera à $\left|\chi^{-1}\left(x_{0}\right)\right|$. L'essentiel de ce travail est consacré à l'étude de ce dernier ensemble dans le cas où $X$ est une surface normale en $x_{0}$.

Définition 2.2. Nous dirons qu'un hyperplan de $\mathbb{C}^{N}$ est général s'il n'est pas une limite d'hyperplans tangents à $X$ en $x_{0}$.

\subsection{Liens entre les limites d'hyperplans tangents et le cône tangent}

Un résultat dû à $H$. Whitney, dans [30] (théorème 22.1), appliqué aux limites d'hyperplans tangents permet de relier le cône tangent à l'ensemble des hyperplans limites d'hyperplans tangents à $X$ en $x_{0}$ :

Si $x$ est un point de $X$ autre que $x_{0}$, on notera $\left(x_{0} x\right)$ la sécante à $X$ en $x_{0}$ passant par $x$.

Théorème 2.3. Si $\left(x_{n}\right)$ est une suite de points non-singuliers de $X$ qui converge vers $x_{0}$, telle que $\lim _{n \rightarrow \infty}\left(x_{0} x_{n}\right)=l$ et si $\left(H_{n}\right)$ est une suite d'hyperplans tangents à $X$ en $x_{n}$ qui converge vers un hyperplan $H$, alors on a $l \subset H$.

En particulier toute génératrice du cône tangent à $X$ en $x_{0}$ est contenue dans une limite d'hyperplans tangents à $X$ en $x_{0}$ et réciproquement, toute limite d'hyperplans tangents contient une génératrice du cône tangent.

Remarque 2.4. Dans le cas d'un germe de courbe, l'ensemble des limites d'hyperplans tangents à $X$ au voisinage de $x_{0}$ est l'ensemble des hyperplans de $\mathbb{C}^{N}$ qui contiennent une droite du cône tangent.

On désignera par $\mathrm{C}_{X, x_{0}}$ le cône tangent à $X$ en $x_{0}$ et par $\left|\mathrm{C}_{X, x_{0}}\right|$ l'ensemble soujaçant.

Dans [13] (théorème 1.2.1), D. T. Là démontre un résultat dont le théorème suivant est une conséquence directe:

Théorème 2.5. Soit $\left(X, x_{0}\right)$ un germe d'espace analytique équidimensionnel réduit. Tout hyperplan tangent au cône tangent $\left|\mathrm{C}_{X, x_{0}}\right|$ le long d'une génératrice est une limite d'hyperplans tangents à $X$ en $x_{0}$.

Voir le paragraphe suivant pour la tangence à un cône le long d'une génératrice. En général, cette inclusion n'est pas une égalité comme le montre l'exemple 
suivant:

Exemple 2.6. L'hypersurface $\mathbf{S}$ de $\mathbb{C}^{3}$ définie par l'équation $x^{2}+y^{2}+z^{3}=0$ a une singularité isolée en 0 . Le cône $\mathrm{C}_{\mathrm{S}, 0}$ est défini par l'équation $x^{2}+y^{2}=0$; c'est la réunion de deux plans. Chacun de ces deux plans est une limite de plans tangents à $\mathrm{S}$ à l'origine. Cependant, tout plan de $\mathbb{C}^{3}$ d'équation $a x+b y=0$; $a, b \in \mathbb{C}$, est aussi une limite d'hyperplans tangents à $\mathrm{S}$ au voisinage de 0 .

\subsection{Cas d'un cône de dimension 2}

Avant de traiter le cas des surfaces normales, nous allons étudier l'ensemble des limites d'hyperplans tangents à un cône de dimension 2 en son sommet. La raison en est que le théorème 2.5 fait intervenir l'ensemble des hyperplans tangents au cône tangent dans l'étude des limites d'hyperplans tangents à un espace donné.

Soit $\left(X, x_{0}\right)$ un cône de dimension 2 réduit de sommet $x_{0}$ que l'on supposera contenu dans $\left(\mathbb{C}^{N}, x_{0}\right)$. L'étude des espaces tangents à $\left(X, x_{0}\right)$ en $x_{0}$ est simplifiée par le fait qu'elle se ramène à l'étude des espaces tangents à la courbe projective $\operatorname{proj} X$.

En effet, l'homogénéité des polynômes définissant le cône $\left(X, x_{0}\right)$ implique qu'un point $x$ du cône différent de $x_{0}$ est singulier si et seulement si tous les points de la génératrice $\left(x x_{0}\right)$ du cône sont singuliers, ce qui est encore équivalent au fait que le point de la courbe $\operatorname{proj} X$ correspondant à cette génératrice est singulier; nous dirons alors que la génératrice $\left(x x_{0}\right)$ est singulière.

Par abus de langage nous dirons qu'une génératrice du cône est non-singulière quand tous ses points autres que le sommet du cône sont non-singuliers; ce qui equivaut à dire que le point lui correspondant dans la courbe $\operatorname{proj} X$ est nonsingulier.

Si une génératrice $l$ du cône est non-singulière, alors un hyperplan $H$ de $\mathbb{C}^{N}$ est tangent au cône en un point de $l\left(\neq x_{0}\right)$ si et seulement s'il est tangent au cône en tout point de $l \quad\left(\neq x_{0}\right)$; ce qui équivaut à dire que l'hyperplan projectif $\operatorname{proj} H$ contient la droite tangente à la courbe projective $\operatorname{proj} X$ au point correspondant à la génératrice $l$. On dira alors que cet hyperplan est tangent au cône le long de la génératrice $l$.

Par extension au cas des génératrices singulières du cône $\left(X, x_{0}\right)$, on dira qu'un hyperplan $H$ est tangent au cône le long d'une génératrice $l$ si l'hyperplan projectif proj $H$ contient la tangente à l'une des branches de la courbe proj $X$ au point $l_{0}$ correspondant à la génératrice $l$.

Dans [22] (1.12), on démontre le résultat suivant:

Proposition 2.7. L'ensemble des limites d'hyperplans tangents à un cône $X$ de dimension 2 en son sommet est l'ensemble des hyperplans tangents au cône le long de ses génératrices (singulières ou non). On notera cet ensemble $\mathcal{T}(X)$. 
Nous appliquerons les resultats de ce paragraphe au cône tangent d'une surface normale en un point singulier.

\subsection{Cas d'une surface normale}

Dans toute la suite de ce travail nous allons nous restreindre au cas où le germe d'espace analytique $\left(X, x_{0}\right)$ est le germe d'une surface normale $\left(\mathrm{S}, x_{0}\right)$ plongée dans $\mathbb{C}^{N}$; ce qui équivaut à dire que le germe de surface $\left(\mathrm{S}, x_{0}\right)$ est à singularité isolée et que l'anneau $\mathcal{O}_{\mathrm{S}, x_{0}}$ est Cohen-Macaulay (cf. [20] VI §2, [32] appendice 6 et [3] théorème 18.15).

Pour simplifier les notations, nous supposerons, quitte à faire un changement de variable, que le point $x_{0}$ est l'origine 0 de $\mathbb{C}^{N}$, et nous désignerons par $\mathrm{S}$ un représentant suffisamment petit du germe $(\mathrm{S}, 0)$.

Le cône tangent $\mathrm{C}_{\mathrm{S}, 0}$ de la surface $\mathrm{S}$ en 0 étant une surface, d'après la proposition 2.7, le théorème 2.5 s'exprime par l'inclusion: $\mathcal{T}\left(\left|\mathrm{C}_{\mathrm{S}, 0}\right|\right) \subset\left|\chi^{-1}(0)\right|$.

Dans [16] (2.1.3), D.T. Là et B. Teissier établissent un résultat qui permet de déterminer l'ensemble des limites d'hyperplans tangents à une surface normale à l'origine. Ce résultat est également contenu dans [5] (page 23).

Théorème 2.8. Il existe un nombre fini de génératrices $l_{1}, \cdots, l_{k}$ de $\left|\mathrm{C}_{\mathrm{S}, 0}\right|$ telles que l'ensemble $\left|\chi^{-1}(0)\right|$ soit la réunion de $\mathcal{T}\left(\left|\mathrm{C}_{\mathrm{S}, 0}\right|\right)$ et de l'ensemble des hyperplans de $\mathbb{C}^{N}$ contenant l'une des génératrices $l_{i}, 1 \leq i \leq k$.

Définition 2.9. Les génératrices du cône tangent citées ci-dessus sont appelées tangentes exceptionnelles de $\mathrm{S}$ en 0.

Une partie importante de ce travail consiste à caractériser puis déterminer les tangentes exceptionnelles d'une surface normale.

\section{Projections linéaires}

Dans cette section, nous étudions le comportement des limites d'hyperplans tangents à une surface normale sous l'effet d'une projection générique sur un plan complexe.

Une projection linéaire $p_{\mathrm{L}}: \mathbb{C}^{N} \rightarrow \mathbb{C}^{2}$, qui a pour noyau un $(N-2)$-plan L, induit un morphisme $\pi_{\mathrm{L}}: \mathrm{S} \rightarrow U \subset \mathbb{C}^{2}$.

Nous dirons que la projection $\pi_{\mathrm{L}}: \mathrm{S} \rightarrow U$ est générique si le morphisme $\pi_{\mathrm{L}}$ est fini et son degré $\operatorname{deg}_{0} \pi_{\mathrm{L}}$ en 0 est égal à la multiplicité $m(\mathrm{~S}, 0)$ de la surface $\mathrm{S}$ en 0 , qui est la multiplicité de l'idéal maximal de l'anneau local $\mathcal{O}_{\mathrm{S}, 0}$.

D'après [24] (I.5.2), on a: 
Proposition 3.1. Une projection linéaire $p_{\mathrm{L}}: \mathbb{C}^{N} \rightarrow \mathbb{C}^{2}$ induit sur $\mathrm{S}$ une projection générique si et seulement si le noyau $\mathrm{L}$ de $p_{\mathrm{L}}$ ne contient aucune génératrice du cône tangent à $\mathrm{S}$ en 0; i.e. $\mathrm{L} \cap\left|\mathrm{C}_{\mathrm{S}, 0}\right|=\{0\}$.

\subsection{Lieu critique et discriminant}

Considérons un morphisme $\pi: \mathrm{S} \rightarrow U$ d'une surface normale sur un ouvert de $\mathbb{C}^{2}$.

Nous définissons le lieu critique de la projection $\pi$ comme étant l'ensemble réduit sous-jacent à l'adhérence dans $\mathrm{S}$ du lieu critique de la restriction de $\pi$ au lieu non-singulier de $\mathrm{S}$. Nous le noterons $\mathrm{C}(\pi)$; c'est une courbe réduite.

Quand la projection $\pi$ est un morphisme fini nous définissons le lieu discriminant de $\pi$ comme étant l'ensemble réduit sous-jacent à l'image par $\pi$ du lieu critique $\mathrm{C}(\pi)$; nous le noterons $\left|\Delta_{\pi}\right|$. C'est une courbe réduite dans l'ouvert $U$ de $\mathbb{C}^{2}$.

Considérons une situation locale, où $\mathrm{S}$ est un représentant d'un germe de surface normale et $U$ est un voisinage ouvert de l'origine dans $\mathbb{C}^{2}$. Un point générique, $x$, d'une composante analytiquement irréductible du lieu discriminant est affecté d'une multiplicité que l'on calcule comme suit: La restriction de $\pi$, au dessus d'un voisinage de $x$, à l'image réciproque par $\pi$ d'une droite transverse à la composante irréductible contenant $x$ est un morphisme fini entre deux courbes non-singulières. Le point $x$ est une valeur critique pour ce morphisme. Si l'on note $\nu_{y}$ l'indice de ramification de ce morphisme en un point $y$ au-dessus de $x$ alors la multiplicité affectée à $x$ est définie par $m_{x}=\Sigma_{y \in \pi^{-1}(x)}\left(\nu_{y}-1\right.$ ) (voir [7] p. 217). Quand la surface $\mathrm{S}$ et l'ouvert $U$ sont suffisamment petits, la semicontinuité du degré de la projection implique que la multiplicité $m_{x}$ est la même en tout point de la branche donnée, différent de l'origine.

Le lieu discriminant de la projection $\pi$ étant une hypersurface dans $U$, il est défini par une fonction $f$. Notons $f_{i}, i=1, \cdots, r$ les composantes analytiquement irréductibles de $f$ au voisinage de l'origine et $m_{i}$ la multiplicité affectée aux points génériques de chacune de ces composantes.

Définition 3.2. Le discriminant de $\pi$ est l'hypersurface de l'ouvert $U$ définie par la fonction $\mathrm{F}=f_{1}^{m_{1}} \ldots f_{r}^{m_{r}}$. On le notera $\Delta_{\pi}$.

Le discriminant défini ci-dessus est la clôture schématique du discriminant usuel de la restriction de la projection au lieu non-singulier de la surface.

Le lieu discriminant est l'ensemble sous-jacent au discriminant.

Dans [28] ( $\S \S 1$ et 2), B. Teissier définit l'espace discriminant en terme d'idéaux de Fitting. La définition que l'on donne ne permet pas d'établir l'invariance par changement de base, cependant elle assure l'inexistence de composantes immergées. 
Dans le cas où la surface $\mathrm{S}$ est une intersection complète ces deux définitions sont équivalentes (voir [27] III. proposition 4).

Cette modification de la définition du discriminant nous permet d'énoncer le lemme 4.4 dans la section suivante.

\subsection{Un lemme clé}

Nous donnons maintenant la caractérisation suivante des hyperplans généraux, en rappelant qu'un hyperplan de $\mathbb{C}^{N}$ est général s'il n'est pas une limite d'hyperplans tangents.

Théorème 3.3. Un hyperplan $H$ de $\mathbb{C}^{N}$ est général si et seulement s'il existe un $(N-2)$-plan $\mathrm{L} \subset H$ tel que $\mathrm{L} \cap\left|\mathrm{C}_{\mathrm{S}, 0}\right|=\{0\}$ et $\pi_{\mathrm{L}}(H \cap \mathrm{S})$ ne soit pas une droite du cône tangent $\mathrm{C}_{\Delta_{\mathrm{L}}, 0}$ du discriminant $\Delta_{\mathrm{L}}$ de $\pi_{\mathrm{L}}$ en 0 , où $\pi_{\mathrm{L}}$ est induit par la projection linéaire de noyau $\mathrm{L}$.

Ce théorème est à la source des principaux résultats énoncés dans ce travail.

Démonstration: Nous suivons une démarche similaire à celle de D. T. Là dans [13] (lemme 2.3.1).

Commençons par prouver qu'un hyperplan $H$ de $\mathbb{C}^{N}$ qui contient un $(N-2)$ plan $\mathrm{L}$ vérifiant $\mathrm{L} \cap\left|\mathrm{C}_{\mathrm{S}, 0}\right|=\{0\}$ et tel que $\pi_{\mathrm{L}}(H \cap \mathrm{S})$ soit une droite du cône tangent, $\mathrm{C}_{\Delta_{\mathrm{L}}, 0}$, du discriminant $\Delta_{\mathrm{L}}$ en 0 est une limite d'hyperplans tangents à $\mathrm{S}$ en 0.

Précisons que puisque le $(N-2)$-plan L est un hyperplan de $H$ et que pour un choix convenable du représentant $\mathrm{S}$ et de l'ouvert $U$ la projection $\pi_{\mathrm{L}}: \mathrm{S} \rightarrow U$ est finie et surjective, l'image $\pi_{\mathrm{L}}(H \cap \mathrm{S})$ n'est autre que la trace sur l'ouvert $U$ de l'image de $H$ par la projection $p_{\mathrm{L}}: \mathbb{C}^{N} \rightarrow \mathbb{C}^{2}$ qui est une droite $\mathrm{D}$ de $\mathbb{C}^{2}$.

La droite $\mathrm{D}$ étant contenue dans le cône tangent réduit $\left|\mathrm{C}_{\Delta_{\mathrm{L}}, 0}\right|$, il existe une suite de droites $\left(\mathrm{T}_{n}\right)$, tangentes au lieu discriminant $\left|\Delta_{\mathrm{L}}\right|$ en des points nonsinguliers $y_{n}$ et qui converge vers D. Il existe une suite de points non-singuliers $\left(x_{n}\right)$ de $\mathrm{S}$ contenue dans le lieu critique de $\pi_{\mathrm{L}}$ telle que pour tout $n, \pi_{\mathrm{L}}\left(x_{n}\right)=$ $y_{n}$. Notons $\mathrm{T}_{x_{n}} \mathrm{~S}$ l'espace tangent à $\mathrm{S}$ en $x_{n}$. Prouvons que $p_{\mathrm{L}}\left(\mathrm{T}_{x_{n}} \mathrm{~S}\right)=\mathrm{T}_{n}$ :

La projection $\pi_{\mathrm{L}}$ est de rang $\leq 1$ en tout point du lieu critique, on a donc $\operatorname{dimp}_{\mathrm{L}}\left(\mathrm{T}_{x_{n}} \mathrm{~S}\right) \leq 1$. Par ailleurs, d'après le théorème de Bertini (cf. [2] théorème 20.1), en choisissant un représentant suffisamment petit du germe (S, 0), la restriction du morphisme $\pi_{\mathrm{L}}$ à son lieu critique est sans point critique sauf éventuellement à l'origine. Ainsi, le morphisme $\pi_{\mathrm{L}}$ est de rang 1 en tout point de son lieu critique autre que l'origine et donc $\operatorname{dim}_{\mathrm{L}}\left(\mathrm{T}_{x_{n}} \mathrm{~S}\right)=1$. D'où l'égalité: $p_{\mathrm{L}}\left(\mathrm{T}_{x_{n}} \mathrm{~S}\right)=\mathrm{T}_{n}$.

Notons $H_{n}$ l'hyperplan $p_{\mathrm{L}}^{-1}\left(\mathrm{~T}_{n}\right)$; c'est un hyperplan tangent à $\mathrm{S}$ en $x_{n}$ puisqu'il contient le plan tangent $\mathrm{T}_{x_{n}} \mathrm{~S}$. Quitte à en extraire une sous-suite, on peut supposer que la suite $\left(H_{n}\right)$ converge dans $\check{\mathbb{P}}^{N-1}$ vers un hyperplan que l'on 
notera $H_{\infty}$. On a alors:

$$
p_{\mathrm{L}}\left(H_{\infty}\right)=p_{\mathrm{L}}\left(\lim _{n \rightarrow \infty} H_{n}\right)=\lim _{n \rightarrow \infty} p_{\mathrm{L}}\left(H_{n}\right)=\lim _{n \rightarrow \infty} \mathrm{T}_{n}=\mathrm{D}=p_{\mathrm{L}}(H) .
$$

Les deux hyperplans $H$ et $H_{\infty}$ sont donc égaux; et par suite l'hyperplan $H$ est une limite d'hyperplans tangents à la surface $\mathrm{S}$ en 0 .

D'autre part, si l'hyperplan $H$ ne contient aucune composante irréductible de $\left|\mathrm{C}_{\mathrm{S}, 0}\right|$ alors l'intersection des espaces projectifs $\operatorname{proj} H$ et $\operatorname{proj}\left|\mathrm{C}_{\mathrm{S}, 0}\right|$ est un nombre fini de points de $\mathbb{P}^{N-1}$. Par conséquent, un hyperplan général L de $H$ n'a aucune droite en commun avec $\left|\mathrm{C}_{\mathrm{S}, 0}\right|$ et donc $\mathrm{L} \cap\left|\mathrm{C}_{\mathrm{S}, 0}\right|=\{0\}$.

Ainsi, si pour tout hyperplan $\mathrm{L}$ de $H$ l'intersection $\mathrm{L} \cap\left|\mathrm{C}_{\mathrm{S}, 0}\right|$ n'est pas réduite à $\{0\}$, l'hyperplan $H$ contient une composante irréductible du cône tangent. D'après le théorème 2.8 , l'hyperplan $H$ est une limite d'hyperplans tangents à $\mathrm{S}$ au voisinage de 0 .

Ceci achève la preuve d'une implication, à savoir que si un hyperplan $H$ est général alors il contient un $(N-2)$-plan $\mathrm{L}$ vérifiant $\mathrm{L} \cap\left|\mathrm{C}_{\mathrm{S}, 0}\right|=\{0\}$ et tel que $\pi_{\mathrm{L}}(H \cap \mathrm{S})$ ne soit pas une droite du cône $\mathrm{C}_{\Delta_{\mathrm{L}}, 0}$.

Réciproquement, considérons un hyperplan $H_{0}$ de $\mathbb{C}^{N}$ qui est une limite d'hyperplans tangents à la surface $\mathrm{S}$ en 0 .

Notons $\lambda: \mathrm{C}\left(\mathrm{S}, \mathbb{C}^{N}\right) \rightarrow \check{\mathbb{P}}^{N-1}$ le morphisme induit sur l'espace conormal de $\mathrm{S}$ dans $\mathbb{C}^{N}$ par la seconde projection de $\mathrm{S} \times \check{\mathbb{P}}^{N-1}$. Nous noterons $\left(0, H_{0}\right)$ un point de $\mathrm{C}\left(\mathrm{S}, \mathbb{C}^{N}\right)$ au-dessus de $H_{0} \in \check{\mathbb{P}}^{N-1}$ et

$$
\lambda_{0}:\left(\mathrm{C}\left(\mathrm{S}, \mathbb{C}^{N}\right),\left(0, H_{0}\right)\right) \rightarrow\left(\check{\mathbb{P}}^{N-1}, H_{0}\right)
$$

le germe de morphisme induit par $\lambda$ au voisinage de $\left(0, H_{0}\right)$.

Remarquons que puisque $\left(0, H_{0}\right) \in \lambda_{0}^{-1}\left(H_{0}\right)$ alors cet ensemble est non-vide.

L'ensemble $\lambda_{0}^{-1}\left(H_{0}\right)$ est l'ensemble des couples $\left(x, H_{0}\right)$ voisins de $\left(0, H_{0}\right)$ dans $\mathrm{C}\left(\mathrm{S}, \mathbb{C}^{N}\right)$ avec $x \in \mathrm{S}$ et $H_{0}$ est tangent ou limite d'hyperplans tangents à $\mathrm{S}$ en $x$.

Trois cas se présentent donc: $\operatorname{dim} \lambda_{0}^{-1}\left(H_{0}\right)=2,1$, ou 0 .

Les deux premiers cas, sont des situations identiques à celles de [13] (lemme 2.3.1). Dans le premier cas, tous les hyperplans de $H_{0}$ ont une génératrice en commun avec le cône tangent $\mathrm{C}_{\mathrm{S}, 0}$. Dans le second, si $\mathrm{L}$ est un hyperplan de $H_{0}$ vérifiant $\mathrm{L} \cap\left|\mathrm{C}_{\mathrm{S}, 0}\right|=\{0\}$ alors la droite contenant l'image $\pi_{\mathrm{L}}\left(H_{0} \cap \mathrm{S}\right)$, de la section $H_{0} \cap \mathrm{S}$, est une droite du cône tangent au discriminant $\Delta_{\mathrm{L}}$ en 0 .

Dans le troisième cas, le morphisme $\lambda_{0}$ est fini. Soit L un $(N-2)$-plan, hyperplan dans $H_{0}$. Notons $\mathcal{C}_{0}$ l'ensemble des hyperplans de $\mathbb{C}^{N}$ contenant ce $(N-2)$-plan L. L'ensemble $\mathcal{C}_{0}$ est un sous-espace de $\check{\mathbb{P}}^{N-1}$ de dimension 1. Rappelons que $\chi: \mathrm{C}\left(\mathrm{S}, \mathbb{C}^{N}\right) \rightarrow \mathrm{S}$ désigne le morphisme conormal de $\mathrm{S}$ dans $\mathbb{C}^{N}$. Notons $\mathcal{C}=\chi\left(\lambda_{0}^{-1}\left(\mathcal{C}_{0}\right)\right)$. Montrons que $\operatorname{dim}(\mathcal{C})=1$ :

Tout d'abord, puisque $\lambda_{0}$ est fini, $\operatorname{dim}\left(\lambda_{0}^{-1}\left(\mathcal{C}_{0}\right)\right)=1$. Le morphisme conormal $\chi$ étant propre, l'espace $\mathcal{C}$ est un sous-espace analytique de $\mathrm{S}$ de dimension 1 ou 0 . Si $\operatorname{dim}(\mathcal{C})=0$, on aura $\mathcal{C}=\{0\}$, donc $\lambda_{0}^{-1}\left(\mathcal{C}_{0}\right)$ est l'ensemble des couples $(0, H)$, 
où $H$ est un hyperplan de $\mathbb{C}^{N}$ qui contient L et qui est une limite d'hyperplans tangents à $\mathrm{S}$ en 0 . Autrement dit, tout hyperplan de $\mathbb{C}^{N}$ contenant $\mathrm{L}$ est une limite d'hyperplans tangents à la surface $\mathrm{S}$ en 0 . Si $\mathrm{L} \cap\left|\mathrm{C}_{\mathrm{S}, 0}\right|=\{0\}$, il existe des hyperplans de $\mathbb{C}^{N}$ qui contiennent $\mathrm{L}$ et qui ne sont pas des limites d'hyperplans tangents. Donc la dimension de $\mathcal{C}$ ne peut pas être 0 . Donc le sous-espace $\mathcal{C}$ de $\mathrm{S}$ est de dimension 1.

La courbe $\mathcal{C}$ est une composante du lieu critique de la projection $\pi_{\mathrm{L}}$. En effet, en tout point de $\mathcal{C}$ non-singulier pour $\mathrm{S}$, l'espace tangent à $\mathrm{S}$ est contenu dans un hyperplan contenant $\mathrm{L}$; le noyau $\mathrm{L}$ de la projection va donc couper cet espace tangent de manière excédentaire.

Considérons une suite de points $\left(x_{n}, H_{n}\right)_{n}$ dans $\lambda_{0}^{-1}\left(\mathcal{C}_{0}\right)$ qui converge vers $\left(0, H_{0}\right)$ dans l'espace conormal $\mathrm{C}\left(\mathrm{S}, \mathbb{C}^{N}\right)$. Chaque hyperplan $H_{n}$ est tangent à la surface $\mathrm{S}$ au point $x_{n} \in \mathcal{C}$ et $\mathrm{L} \subset H_{n}$. Par conséquent, l'image $\pi_{\mathrm{L}}\left(H_{n} \cap \mathrm{S}\right)$ est une droite tangente au lieu discriminant $\left|\Delta_{\mathrm{L}}\right|$ de $\pi_{\mathrm{L}}$. Quitte à en extraire une sous-suite, la suite de droites $\pi_{\mathrm{L}}\left(H_{n} \cap \mathrm{S}\right)$ converge vers une droite du cône $\mathrm{C}_{\Delta_{\mathrm{L}}, 0}$. Or $\pi_{\mathrm{L}}\left(H_{n} \cap \mathrm{S}\right)$ converge vers $\pi_{\mathrm{L}}\left(H_{0} \cap \mathrm{S}\right)$. L'image $\pi_{\mathrm{L}}\left(H_{0} \cap \mathrm{S}\right)$ est donc une droite du cône tangent $\mathrm{C}_{\Delta_{\mathrm{L}}, 0}$ du discriminant $\Delta_{\mathrm{L}}$ de $\pi_{\mathrm{L}}$ en 0 .

On a donc démontré la deuxième implication, à savoir que si un hyperplan $H$ contient un $(N-2)$-plan $\mathrm{L}$ verifiant $\mathrm{L} \cap\left|\mathrm{C}_{\mathrm{S}, 0}\right|=\{0\}$ et si l'image $\pi_{\mathrm{L}}(H \cap \mathrm{S})$ n'est pas tangente au discriminant $\Delta_{\mathrm{L}}$, alors $H$ est général.

\section{Minimalité du nombre de Milnor}

Dans le cas d'un germe d'hypersurface de $\mathbb{C}^{N}$ à singularité isolée, B. Teissier démontre dans [27] (I.1.4) qu'un hyperplan de $\mathbb{C}^{N}$ est général si et seulement si sa section avec l'hypersurface est à singularité isolée et son nombre de Milnor est minimum parmi les nombres de Milnor des sections hyperplanes à singularités isolées. Nous nous proposons, dans cette section, de démontrer une condition nécessaire et suffisante analogue et valable dans le cas des germes de surfaces normales.

Dans [18] (§§6 et 7), J. Milnor définit le nombre de Milnor pour des hypersurfaces à singularités isolées. Dans [12], H. Hamm définit aussi un nombre de Milnor pour les intersections complètes à singularités isolées.

Dans le cas des surfaces normales de $\mathbb{C}^{N}$, les sections hyperplanes sont en général des courbes gauches qui ne sont pas des intersections complètes. Cependant, dans [1] (§1.1), il est associé à une singularité de courbe réduite, un nombre $\mu$ qui a des propriétés topologiques analogues à celles du nombre de Milnor et qui coincide avec celui-ci dans le cas des intersections complètes (cf. [1] (lemme 1.1.2) et [10] (appendice 1)).

Considérons un germe de courbe réduite $(\mathcal{C}, 0)$ et notons $n: \overline{\mathcal{C}} \rightarrow(\mathcal{C}, 0)$ sa normalisation. Posons $\delta=\operatorname{dim}_{\mathbb{C}}\left(n_{*} \mathcal{O}_{\overline{\mathcal{C}}}\right)_{0} / \mathcal{O}_{\mathcal{C}, 0}$ et appelons $r$ le nombre de branches du germe $(\mathcal{C}, 0)$. Nous avons une généralisation du nombre de Milnor pour un 
germe de courbe réduite défini de la manière suivante (cf. [1] (1.1.1 et 1.2.1)):

Définition 4.1. On appellera nombre de Milnor du germe de courbe $(\mathcal{C}, 0)$ et on notera $\mu(\mathcal{C}, 0)$ l'entier $2 \delta-r+1$.

Le résultat principal de cette section est le suivant:

Théorème 4.2. Soit $\mathrm{S}$ un représentant d'un germe de surface analytique normal plongé dans $\mathbb{C}^{N}$. Un hyperplan $H$ de $\mathbb{C}^{N}$ ne contenant aucune composante irréductible du cône $\left|\mathrm{C}_{\mathrm{S}, 0}\right|$ est général si et seulement si la section $H \cap \mathrm{S}$ est réduite et $\mu(H \cap \mathrm{S}, 0)$ est minimum parmi les nombres de Milnor en 0 des sections hyperplanes réduites.

Remarquons que dans le cas où l'hyperplan $H$ contient une composante irréductible du cône tangent $\left|\mathrm{C}_{\mathrm{S}, 0}\right|$ alors, d'après le théorème 2.5 , c'est une limite d'hyperplans tangents à $\mathrm{S}$ à l'origine.

D'autre part l'anneau local $\mathcal{O}_{\mathrm{S}, 0}$ des fonctions holomorphes sur S étant CohenMacaulay, les sections hyperplanes de $\mathrm{S}$ qui sont des courbes, sont sans composantes immergées (cf. [32] appendice 6 corollaire 3 ).

Avant de démontrer ce théorème, nous allons énoncer deux lemmes:

Le premier lemme permet de régler le cas des sections hyperplanes qui ont des singularités non-isolées.

Lemme 4.3. Si l'hyperplan $H$ est général alors la section de $H$ avec $\mathrm{S}$ est une courbe réduite.

Démonstration: Considérons un hyperplan $H$ dont la section avec $\mathrm{S}$ n'est pas réduite. Notons $\mathrm{E}$ une composante non-réduite de $H \cap \mathrm{S}$. En tout point $x$ de $\mathrm{E}$ l'hyperplan $H$ n'est pas transverse à $\mathrm{S}$, il contient donc le plan tangent à la surface $\mathrm{S}$ en tout point de $\mathrm{E}$. En prenant une suite de points $\left(x_{n}\right)$ de la composante $\mathrm{E}$ qui converge vers 0 , l'hyperplan $H$ est tangent à $\mathrm{S}$ en tout point $x_{n}$ non-singulier; c'est donc une limite d'hyperplans tangents à $\mathrm{S}$ à l'origine.

Le second lemme permet d'établir un lien entre le nombre de Milnor d'une section hyperplane réduite et la multiplicité d'intersection de son image avec le discriminant d'une projection linéaire générique. Reprenons les notations de la section précédente: $\pi_{\mathrm{L}}: \mathrm{S} \rightarrow U$ est la projection générique induite par un $(N-2)$ plan $\mathrm{L}$ et $\Delta_{\mathrm{L}}$ est son discriminant défini dans 3.2. On a:

Lemme 4.4. Soit $l: \mathbb{C}^{2} \rightarrow \mathbb{C}$ une forme linéaire et soit $\sigma=l \circ \pi_{\mathrm{L}}$. Si la courbe $\sigma^{-1}(0)$ est réduite, la multiplicité d'intersection $\left(\Delta_{\mathrm{L}} \cdot l^{-1}(0)\right)_{0}$ en 0 est égale à $\mu\left(\sigma^{-1}(0), 0\right)+\operatorname{deg}_{0} \pi_{\mathrm{L}}-1$. 
Nous remercions le professeur D.T. Là qui nous a aimablement communiqué une preuve de ce lemme.

Démonstration: Si l'on note $\mathrm{D}$ un disque ouvert dans $\mathbb{C}$ centré en 0 et de rayon suffisamment petit et si l'on choisit un bon représentant $\mathrm{S}$ du germe de surface (S,0) qui, rappelons-le, est normal, le morphisme $\sigma: \mathrm{S} \rightarrow \mathrm{D}$ est une lissification de la courbe $\sigma^{-1}(0)$.

Si l'on note $\chi\left(\sigma^{-1}(t)\right)$ la caractéristique d'Euler de la courbe $\sigma^{-1}(t)$ pour $t \in \mathrm{D}$ et si l'on suppose que la courbe $\sigma^{-1}(0)$ est réduite alors, d'après [1] (corollaire 4.2.3), on a:

$$
\chi\left(\sigma^{-1}(t)\right)=1-\mu\left(\sigma^{-1}(0), 0\right) .
$$

En considérant la restriction de la projection $\pi_{\mathrm{L}}$ à la courbe $\sigma^{-1}(t)$, et en remarquant que le lieu de ramification de cette restriction est l'ensemble des points d'intersection de la courbe $l^{-1}(t)$ avec le discriminant $\Delta_{\mathrm{L}}$ de $\pi_{\mathrm{L}}$, on obtient:

$$
\begin{aligned}
\chi\left(\sigma^{-1}(t)\right)= & \operatorname{deg}_{0} \pi_{\mathrm{L}}\left(1-\#\left(l^{-1}(t) \cap \Delta_{\mathrm{L}}\right)\right) \\
& +\sum_{x_{i} \in l^{-1}(t) \cap \Delta_{\mathrm{L}}}\left(\operatorname{deg}_{0} \pi_{\mathrm{L}}-\sum_{y_{i, j} \in \pi_{\mathrm{L}}^{-1}\left(x_{i}\right)}\left(\operatorname{deg}_{y_{i, j}} \pi_{\mathrm{L}}-1\right)\right) \\
= & \operatorname{deg}_{0} \pi_{\mathrm{L}}-\sum_{x_{i} \in l^{-1}(t) \cap \Delta_{\mathrm{L}}} \sum_{y_{i, j} \in \pi_{\mathrm{L}}-1\left(x_{i}\right)}\left(\operatorname{deg}_{y_{i, j}} \pi_{\mathrm{L}}-1\right) .
\end{aligned}
$$

Par ailleurs, le discriminant $\Delta_{\mathrm{L}}$ étant une hypersurface dans un ouvert de $\mathbb{C}^{2}$, on a:

$$
\left(l^{-1}(0) \cdot \Delta_{\mathrm{L}}\right)_{0}=\sum_{x_{i} \in l^{-1}(t) \cap \Delta_{\mathrm{L}}}\left(l^{-1}(t) \cdot \Delta_{\mathrm{L}}\right)_{x_{i}} .
$$

Or on a:

$$
\left(l^{-1}(t) \cdot \Delta_{\mathrm{L}}\right)_{x_{i}}=\sum_{y_{i, j} \in \pi_{\mathrm{L}}^{-1}\left(x_{i}\right)}\left(\operatorname{deg}_{y_{i, j}} \pi_{\mathrm{L}}-1\right) .
$$

En remplaçant (4.3) dans (4.2) puis dans (4.1) on a:

$$
\chi\left(\sigma^{-1}(t)\right)=\operatorname{deg}_{0} \pi_{\mathrm{L}}-\left(l^{-1}(0) \cdot \Delta_{\mathrm{L}}\right)_{0} .
$$

D'où l'égalité:

$$
\mu\left(\sigma^{-1}(0), 0\right)=1-\operatorname{deg}_{0} \pi_{\mathrm{L}}+\left(l^{-1}(0) \cdot \Delta_{\mathrm{L}}\right)_{0} .
$$

Nous pouvons maintenant donner la démonstation du théorème 4.2:

Démonstration: Précisons que si un hyperplan $H$ de $\mathbb{C}^{N}$ ne contient aucune composante irréductible du cône $\left|\mathrm{C}_{\mathrm{S}, 0}\right|$ alors l'intersection $H \cap\left|\mathrm{C}_{\mathrm{S}, 0}\right|$ est un nombre fini de droites et donc un hyperplan générique $\mathrm{L}$ de $H$ vérifie la condition $\mathrm{L} \cap\left|\mathrm{C}_{\mathrm{S}, 0}\right|=\{0\}$.

Considérons un hyperplan $H$ qui ne contient aucune composante irréductible de $\left|\mathrm{C}_{\mathrm{S}, 0}\right|$, qui soit une limite d'hyperplans tangents à la surface $\mathrm{S}$ en 0 et tel que la section $H \cap \mathrm{S}$ soit réduite. Considérons alors un $(N-2)$-plan $\mathrm{L}$ contenu dans 
$H$ tel que $\mathrm{L} \cap\left|\mathrm{C}_{\mathrm{S}, 0}\right|=\{0\}$ et notons $\pi_{\mathrm{L}}: \mathrm{S} \rightarrow U$ la projection générique définie par $\mathrm{L}$ comme dans la section prédédente.

Pour le màme $(N-2)$-plan $\mathrm{L}$, nous avons vu lors de la preuve du théorème 3.3 , qu'il existe un hyperplan $H^{\prime}$ général contenant L. D'après le lemme 4.3 , la courbe $H^{\prime} \cap \mathrm{S}$ est aussi réduite.

D'après le lemme 4.4, on a:

$$
\text { et } \begin{aligned}
\left(\pi_{\mathrm{L}}(H \cap \mathrm{S}) \cdot \Delta_{\mathrm{L}}\right)_{0} & =\mu(H \cap \mathrm{S}, 0)+\operatorname{deg}_{0} \pi_{\mathrm{L}}-1 \\
\left(\pi_{\mathrm{L}}\left(H^{\prime} \cap \mathrm{S}\right) \cdot \Delta_{\mathrm{L}}\right)_{0} & =\mu\left(H^{\prime} \cap \mathrm{S}, 0\right)+\operatorname{deg}_{0} \pi_{\mathrm{L}}-1
\end{aligned}
$$

D'aprés le théorème 3.3, la droite $\pi_{\mathrm{L}}(H \cap \mathrm{S})$ est une droite du cône tangent à $\Delta_{\mathrm{L}}$ en 0 et la droite $\pi_{\mathrm{L}}\left(H^{\prime} \cap \mathrm{S}\right)$ n'en est pas. Par conséquent d'après [4] (III. $\S 3)$, on a:

$$
\text { et } \begin{aligned}
& \left(\pi_{\mathrm{L}}(H \cap \mathrm{S}) \cdot \Delta_{\mathrm{L}}\right)_{0}>m\left(\Delta_{\mathrm{L}}, 0\right) \\
& \left(\pi_{\mathrm{L}}\left(H^{\prime} \cap \mathrm{S}\right) \cdot \Delta_{\mathrm{L}}\right)_{0}=m\left(\Delta_{\mathrm{L}}, 0\right)
\end{aligned}
$$

Il résulte de (4.4) et (4.5) que:

$$
\mu(H \cap \mathrm{S}, 0)>\mu\left(H^{\prime} \cap \mathrm{S}, 0\right) .
$$

Le nombre de Milnor de $H \cap \mathrm{S}$ en 0 n'est donc pas minimum.

Réciproquement, considérons un hyperplan $H$ de $\mathbb{C}^{N}$ ne contenant aucune composante irréductible de $\left|\mathrm{C}_{\mathrm{S}, 0}\right|$.

Si la courbe $H \cap \mathrm{S}$ n'est pas réduite alors d'après le lemme 4.3, l'hyperplan $H$ est une limite d'hyperplans tangents à $\mathrm{S}$ à l'origine.

Supposons maintenant que la courbe $H \cap \mathrm{S}$ est réduite et le nombre de Milnor $\mu(H \cap \mathrm{S}, 0)$ n'est pas minimum. D'après [1] (théorème 6.1.7), il existe un ouvert dense $\Omega$ de l'ensemble des hyperplans de $\mathbb{C}^{N}$ tel que pour tout $H^{\prime} \in \Omega$ on ait $H^{\prime} \cap \mathrm{S}$ est réduite et $\mu\left(H^{\prime} \cap \mathrm{S}, 0\right)<\mu(H \cap \mathrm{S}, 0)$. L'ensemble des intersections des hyperplans contenus dans $\Omega$ avec l'hyperplan $H$ est un ouvert dense de l'ensemble des hyperplans de $H$. Par ailleurs, puisque l'hyperplan $H$ ne contient aucune composante irréductible de $\left|\mathrm{C}_{\mathrm{S}, 0}\right|$, l'ensemble des hyperplans de $H$ n'ayant aucune génératrice en commun avec le cône $\left|\mathrm{C}_{\mathrm{S}, 0}\right|$ est aussi un ouvert dense de l'ensemble des hyperplans de $H$. Il existe donc un hyperplan $H^{\prime}$ de $\mathbb{C}^{N}$ tel qu'on ait à la fois $H^{\prime} \cap \mathrm{S}$ est une courbe réduite, $\mu\left(H^{\prime} \cap \mathrm{S}, 0\right)<\mu(H \cap \mathrm{S}, 0)$ et si l'on note $\mathrm{L}$ le $(N-2)$-plan de $\mathbb{C}^{N}$ défini par l'intersection $H^{\prime} \cap H$ alors $\mathrm{L} \cap\left|\mathrm{C}_{\mathrm{S}, 0}\right|=\{0\}$.

Si l'on note $\pi_{\mathrm{L}}$ la projection générique définie par $\mathrm{L}$, on a d'après le lemme 4.4 ,

$$
\text { et } \begin{aligned}
\left(\Delta_{\mathrm{L}} \cdot \pi_{\mathrm{L}}(H \cap \mathrm{S})\right)_{0} & =\mu(H \cap \mathrm{S}, 0)+\operatorname{deg}_{0} \pi_{\mathrm{L}}-1 \\
\left(\Delta_{\mathrm{L}} \cdot \pi_{\mathrm{L}}\left(H^{\prime} \cap \mathrm{S}\right)\right)_{0} & =\mu\left(H^{\prime} \cap \mathrm{S}, 0\right)+\operatorname{deg}_{0} \pi_{\mathrm{L}}-1
\end{aligned}
$$

Puisque $\mu\left(H^{\prime} \cap \mathrm{S}, 0\right)<\mu(H \cap \mathrm{S}, 0)$, on a alors

$$
\left(\Delta_{\mathrm{L}} \cdot \pi_{\mathrm{L}}(H \cap \mathrm{S})\right)_{0}>\left(\Delta_{\mathrm{L}} \cdot \pi_{\mathrm{L}}\left(H^{\prime} \cap \mathrm{S}\right)\right)_{0} .
$$


Par conséquent, la droite $\left(\pi_{\mathrm{L}}(H \cap S)\right)$ est une droite du cône tangent au discriminant $\Delta_{\mathrm{L}}$ en 0 . D'après le théorème 3.3 , l'hyperplan $H$ est une limite d'hyperplans tangents à la surface $\mathrm{S}$ à l'origine. Ce qui achève la preuve du théorème 4.2.

Exemple. Considérons la surface normale $\mathbf{S}$ de $\mathbb{C}^{4}$ définie par $\left(x^{2}+y^{3}+z^{2}+\right.$ $\left.t^{4}, 2 x^{2}+3 y^{3}+z^{2}-t^{4}\right)$. Le cône tangent réduit $\left|\mathrm{C}_{\mathrm{S}, 0}\right|$ est le plan de $\mathbb{C}^{4}$ défini par $(x, z)$.

Considérons maintenant le 2-plan $\mathrm{L}$ de $\mathbb{C}^{4}$ défini par $(y, t)$. On a bien la condition de généricité $\mathrm{L} \cap\left|\mathrm{C}_{\mathrm{S}, 0}\right|=\{0\}$. Le discriminant de la projection $\pi_{\mathrm{L}}$ est l'hypersurface $\Delta_{\mathrm{L}}$ de $\mathbb{C}^{2}$ définie par $\left(\left(y^{3}-t^{4}\right)^{2}\left(y^{3}-3 t^{4}\right)^{2}\right)$. On a $m\left(\Delta_{\mathrm{L}}, 0\right)=12$ et $\left|\mathrm{C}_{\Delta_{\mathrm{L}}, 0}\right|$ est la droite de $\mathbb{C}^{2}$ définie par $(y)$. Le degré de la projection $\pi_{\mathrm{L}}$ vaut 4.

Notons $H_{y=0}$ et $H_{t=0}$ les hyperplans de $\mathbb{C}^{4}$ définis respectivement par $(y)$ et par $(t)$. D'après le théorème 3.3 , l'hyperplan $H_{y=0}$ est une limite d'hyperplans tangents à $\mathrm{S}$ en 0 et l'hyperplan $H_{t=0}$ est général.

Les sections hyperplanes $H_{y=0} \cap \mathrm{S}$ et $H_{t=0} \cap \mathrm{S}$ sont définies respectivement par $\left(y, x^{2}-2 t^{4}, z^{2}+3 t^{4}\right)$ et par $\left(t, x^{2}+2 z^{2}, y^{3}-z^{2}\right)$. Dans les deux cas ce sont des intersections complètes définies par des polynômes quasi-homogènes. En appliquant la formule donnée par M. Giusti dans [11] (\$1) pour le calcul du nombre de Milnor dans ce cas, on obtient:

$$
\mu\left(H_{y=0} \cap \mathrm{S}, 0\right)=13 \text { et } \mu\left(H_{t=0} \cap \mathrm{S}, 0\right)=9 .
$$

Par ailleurs, si l'on note $\mu_{\text {min }}=m\left(\Delta_{\mathrm{L}}, 0\right)+m(\mathrm{~S}, 0)-1$, qui dans ce cas vaut $12-4+1=9$, alors d'après le théorème 4.2 , on doit avoir $\mu\left(H_{t=0} \cap \mathrm{S}, 0\right)=\mu_{\text {min }}$ et $\mu\left(H_{y=0} \cap \mathrm{S}, 0\right)>\mu_{\text {min }}$, ce qui est le cas d'après le calcul des nombres de Milnor.

Remarquons que l'hyperplan $H_{y=0}$ n'est pas tangent au cône $\left|\mathrm{C}_{\mathrm{S}, 0}\right|$. D'après le théorème 2.8, l'hyperplan $H_{y=0}$ contient donc une tangente exceptionnelle de la surface $\mathrm{S}$ en 0 . Dans ce cas, la droite définie par $(x, y, z)$ est cette tangente exceptionnelle de $\mathrm{S}$ en 0.

\section{Tangentes exceptionnelles}

Rappelons que d'après le théorème 2.8 , la détermination de l'ensemble des limites d'hyperplans tangents à une surface normale en un point singulier passe par la détermination des tangentes exceptionnelles de cette surface en ce point singulier.

Nous allons commencer par donner une caractérisation des tangentes exceptionnelles d'un germe de surface normale en terme de résolution simultanée faible des familles des sections hyperplanes. 


\subsection{Résolution simultanée faible}

Considérons un germe de morphisme plat d'espaces analytiques $f_{x_{0}}:\left(X, x_{0}\right) \rightarrow$ $\left(\mathrm{D}, d_{0}\right)$ où $\left(X, x_{0}\right)$ est le germe d'une surface et $\left(\mathrm{D}, d_{0}\right)$ celui d'une courbe non-singulière. Notons $f: X \rightarrow \mathrm{D}$ un représentant suffisamment petit du germe $f_{x_{0}}, n: \tilde{X} \rightarrow X$ la normalisation de la surface $X$ et $\tilde{f}: \tilde{X} \rightarrow$ D le morphisme composé $f \circ n$.

La normalisation en famille et la résolution simultanée faible de $f: X \rightarrow \mathrm{D}$ sont définies comme suit (cf. [26] §1 et [1] 4.1.5):

Définition 5.1. La famille de courbes $f: X \rightarrow \mathrm{D}$ a une normalisation en famille si pour tout $d \in \mathrm{D}$ la courbe $\tilde{f}^{-1}(d)$ est non-singulière.

Si de plus $f$ a une section holomorphe $\sigma: \mathrm{D} \rightarrow X$ telle que $\tilde{f}^{-1}(d)-\{\sigma(d)\}$ soit non-singulière pour tout $d \in \mathrm{D}$ et $\left|n^{-1}(\sigma(\mathrm{D}))\right| \stackrel{\simeq}{=} \times\left|n^{-1}\left(\sigma\left(d_{0}\right)\right)\right|$ alors on dit que le morphisme $f$ a une résolution simultanée faible en $x_{0}$ le long de $\sigma(\mathrm{D})$.

Dans le cas où les fibres du morphisme $f$ sont des courbes planes, la résolution simultanée faible est caractérisée par l'une des nombreuses notions équivalentes d'équisingularité d'une surface en un point le long d'une courbe (cf. [16] \$1.2). Certaines de ces équivalences ne sont plus valables dans le cas des courbes gauches. Cependant nous disposons des équivalences suivantes ([26] théorème 1.3 .2 et [1] théorème 5.2.2):

Théorème 5.2. Soit $f: X \rightarrow \mathrm{D}$ comme ci-dessus.

a) Le morphisme $f$ a une normalisation en famille si et seulement si pour tout $d \in \mathrm{D}$ la courbe $f^{-1}(d)$ est réduite et $\delta\left(f^{-1}(d)\right)=\delta\left(f^{-1}\left(d_{0}\right)\right)$, où $\delta\left(f^{-1}(d)\right)=$ $\sum_{x \in f^{-1}(d)} \delta\left(f^{-1}(d), x\right)$, l'invariant $\delta$ étant celui introduit pour la définition 4.1.

b) Le morphisme $f$ a une résolution simultanée faible si et seulement si l'une des propriétés équivalentes suivantes est vérifiée:

(i) Le morphisme $f$ a une normalisation en famille et il existe une section $\sigma: \mathrm{D} \rightarrow X$ telle que le nombre de branches, $r\left(f^{-1}(d), \sigma(d)\right)$, de $f^{-1}(d)$ au point $\sigma(d)$ soit constant pour tout $d \in \mathrm{D}$.

(ii) Le morphisme $f$ est à fibres réduites et a une section $\sigma: \mathrm{D} \rightarrow X$ telle que le nombre de Milnor $\mu\left(f^{-1}(d), \sigma(d)\right)$ soit constant pour tout $d \in \mathrm{D}$.

\subsection{Caractérisations des tangentes exceptionnelles}

Nous allons maintenant étudier l'existence d'une résolution simultanée faible dans le cas des familles de sections hyperplanes d'une surface normale.

Considérons un $(N-2)$-plan L de $\mathbb{C}^{N}$ tel que $\mathrm{L} \cap\left|\mathrm{C}_{\mathrm{S}, 0}\right|=\{0\}$. La projection générique $\pi_{\mathrm{L}}: \mathrm{S} \rightarrow U \subset \mathbb{C}^{2}$ est définie par deux fonctions holomorphes $f$ et $g$ 
contenues dans l'idéal maximal $\mathfrak{m}$ de l'anneau local $\mathcal{O}_{\mathrm{S}, 0}$. La généricité de $\pi_{\mathrm{L}}$ fait que la paire $(f, g)$ est une suite régulière de l'anneau $\mathcal{O}_{\mathrm{S}, 0}$. Considérons l'éclatement $e_{\mathrm{L}}: \mathrm{S}_{\mathrm{L}}^{\prime} \rightarrow \mathrm{S}$ de l'idéal primaire $(f, g) \mathcal{O}_{\mathrm{S}, 0}$. Nous avons alors le diagramme commutatif suivant:

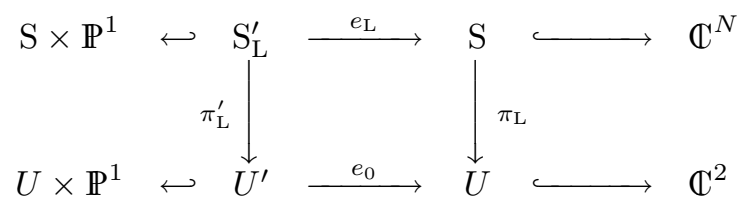

où $e_{0}$ est l'éclatement de l'origine dans $U$ et $\pi_{\mathrm{L}}^{\prime}$ est donné par la propriété universelle de l'éclatement.

La surface $\mathrm{S}_{\mathrm{L}}^{\prime}$ est définie dans $\mathrm{S} \times \mathbb{P}^{1}$ par l'idéal $\left(f \mathrm{~T}_{2}-g \mathrm{~T}_{1}\right)$ de $\mathcal{O}_{\mathrm{S}, 0}\left[\mathrm{~T}_{1}, \mathrm{~T}_{2}\right]$, où $\left(\mathrm{T}_{1}: \mathrm{T}_{2}\right)$ est un système de coordonnées homogènes dans $\mathbb{P}^{1}$ (cf. [3] 17.14). Par unicité du morphisme $\pi_{\mathrm{L}}^{\prime}$ donné par la propriété universelle de l'éclatement, $\pi_{\mathrm{L}}^{\prime}$ est le morphisme induit sur $\mathrm{S}_{\mathrm{L}}^{\prime}$ par la projection $\left(\pi_{\mathrm{L}}, \mathrm{I} d_{\mathbb{P}^{1}}\right): \mathrm{S} \times \mathbb{P}^{1} \rightarrow U \times \mathbb{P}^{1}$. Le morphisme $\pi_{\mathrm{L}}^{\prime}$ est un morphisme fini.

Considérons la projection $p_{r_{2}}: U^{\prime} \rightarrow \mathbb{P}^{1}$, et notons $f: \mathrm{S}_{\mathrm{L}}^{\prime} \rightarrow \mathbb{P}^{1}$ le morphisme composé $p_{r_{2}} \circ \pi_{\mathrm{L}}^{\prime}$. Les fibres du morphisme $f$ sont exactement les transformées strictes par $e_{\mathrm{L}}$ des sections hyperplanes de la surface $\mathrm{S}$ définies par les hyperplans de $\mathbb{C}^{N}$ qui contiennent le $(N-2)$-plan L. Une section hyperplane de $\mathrm{S}$ définie par un hyperplan contenant le $(N-2)$-plan L étant isomorphe à sa transformée stricte par l'éclatement $e_{\mathrm{L}}$ on peut voir le morphisme $f: \mathrm{S}_{\mathrm{L}}^{\prime} \rightarrow \mathbb{P}^{1}$ comme la famille des sections hyperplanes de $\mathrm{S}$ définies par les hyperplans de $\mathbb{C}^{N}$ contenant L.

D'autre part, la surface $S$ étant normale, l'espace $S \times \mathbb{P}^{1}$ est Cohen-Macaulay au voisinage de chacun de ses points. La surface $\mathrm{S}_{\mathrm{L}}^{\prime}$ étant une hypersurface dans $\mathrm{S} \times \mathbb{P}^{1}$, son anneau de fonctions holomorphes est donc localement Cohen-Macaulay. Il en résulte que le morphisme $f: \mathrm{S}_{\mathrm{L}}^{\prime} \rightarrow \mathbb{P}^{1}$ est plat.

On fera alors une étude locale de l'existence de résolution simultanée faible de la famille de sections hyperplanes $f: \mathrm{S}_{\mathrm{L}}^{\prime} \rightarrow \mathbb{P}^{1}$ en chacun des points du diviseur exceptionnel de $\mathrm{S}_{\mathrm{L}}^{\prime}$.

5.3. Considérons un point $x_{0}$ de la surface $\mathrm{S}_{\mathrm{L}}^{\prime}$ appartenant à la fibre exceptionnelle de l'éclatement $e_{\mathrm{L}}$. Notons $X_{\mathrm{L}}$ un représentant assez petit du germe de surface $\left(\mathrm{S}_{\mathrm{L}}^{\prime}, x_{0}\right), \mathrm{D}_{\mathrm{L}}$ son image par le morphisme $f$, et $f_{\mathrm{L}}: X_{\mathrm{L}} \rightarrow \mathrm{D}_{\mathrm{L}} \subset \mathbb{P}^{1}$ le morphisme induit par $f$.

Précisons que le morphisme $f_{\mathrm{L}}: X_{\mathrm{L}} \rightarrow \mathrm{D}_{\mathrm{L}}$ ainsi construit, a une section naturelle $\sigma: \mathrm{D}_{\mathrm{L}} \rightarrow X_{\mathrm{L}}$ qui à tout point $d \in \mathrm{D}_{\mathrm{L}}$ associe le point $\sigma(d) \in X_{\mathrm{L}}$ défini comme étant l'unique point d'intersection de la fibre $f_{\mathrm{L}}^{-1}(d)$ avec le diviseur exceptionnel de l'éclatement $e_{\mathrm{L}}$ dans $X_{\mathrm{L}}$.

Théorème 5.4. Soit $H$ un hyperplan de $\mathbb{C}^{N}$ ne contenant aucune composante 
irréductible du cône $\left|\mathrm{C}_{\mathrm{S}, 0}\right|$. H est général si et seulement si pour tout hyperplan $\mathrm{L}$ de $H$ vérifiant $\mathrm{L} \cap\left|\mathrm{C}_{\mathrm{S}, 0}\right|=\{0\}$ on a: si l'on note $x_{0}$ le point exceptionnel de la transformée stricte, par $e_{\mathrm{L}}$, de la section $H \cap \mathrm{S}$ et $f_{\mathrm{L}}: X_{\mathrm{L}} \rightarrow \mathrm{D}_{\mathrm{L}}$ le morphisme construit, comme dans 5.3, autour de $x_{0}$, alors $f_{\mathrm{L}}$ a une résolution simultanée faible le long de $\sigma\left(\mathrm{D}_{\mathrm{L}}\right)$.

Démonstration: Soit $H$ un hyperplan de $\mathbb{C}^{N}$ ne contenant aucune composante irréductible du cône $\left|\mathrm{C}_{\mathrm{S}, 0}\right|$.

Supposons que l'hyperplan $H$ est général. Soit L un $(N-2)$-plan de $\mathbb{C}^{N}$ contenu dans $H$ et tel que $\mathrm{L} \cap\left|\mathrm{C}_{\mathrm{S}, 0}\right|=\{0\}$. Notons $x_{0}$ le point d'intersection de la transformée stricte de $H \cap \mathrm{S}$ par l'éclatement $e_{\mathrm{L}}$ avec le diviseur exceptionnel de $e_{\mathrm{L}}$. Rappelons que l'ensemble des hyperplans généraux de $\mathbb{C}^{N}$ contenant $\mathrm{L}$ est un ouvert dense de l'ensemble des hyperplans de $\mathbb{C}^{N}$ contenant L. On peut donc choisir un représentant suffisamment petit $X_{\mathrm{L}}$ du germe $\left(\mathrm{S}_{\mathrm{L}}^{\prime}, x_{0}\right)$ de sorte que toutes les sections hyperplanes de $\mathrm{S}$ dont les transformées strictes par $e_{\mathrm{L}}$ coupent le diviseur exceptionnel de $e_{\mathrm{L}}$ dans $X_{\mathrm{L}}$ soient définies par des hyperplans généraux de $\mathbb{C}^{N}$. Considérons alors le morphisme $f_{\mathrm{L}}: X_{\mathrm{L}} \rightarrow \mathrm{D}_{\mathrm{L}}$ défini autour de $x_{0}$ comme dans 5.3.

Rappelons que chaque fibre du morphisme $f: S_{L}^{\prime} \rightarrow \mathbb{P}^{1}$ est isomorphe à la section hyperplane de $\mathrm{S}$ dont elle est la transformée stricte par l'éclatement $e_{\mathrm{L}}$. Il en résulte que si une section $H^{\prime} \cap \mathrm{S}$ réduite a comme transformée stricte la fibre $f^{-1}(d), d \in \mathbb{P}^{1}$, alors on a:

$$
\mu\left(f^{-1}(d), \sigma(d)\right)=\mu\left(H^{\prime} \cap \mathrm{S}, 0\right) .
$$

Dans le voisinage $X_{\mathrm{L}}$ de $x_{0}$, les fibres du morphisme $f_{\mathrm{L}}$ sont toutes les transformées strictes de sections hyperplanes définies par des hyperplans généraux de $\mathbb{C}^{N}$. D'après le théorème 4.2 , pour tout $d \in \mathrm{D}_{\mathrm{L}}$, les fibres $f_{\mathrm{L}}^{-1}(d)$ sont réduites et

$$
\mu\left(f_{\mathrm{L}}^{-1}(d), \sigma(d)\right)=\mu(H \cap \mathrm{S}, 0)
$$

et vaut la valeur minimale $\mu_{\min }$ parmi les nombres de Milnor en 0 des sections hyperplanes réduites de $\mathrm{S}$. D'après le théorème 5.2 , le morphisme $f_{\mathrm{L}}: X_{\mathrm{L}} \rightarrow \mathrm{D}_{\mathrm{L}}$ a une résolution simultanée faible le long de la section $\sigma\left(\mathrm{D}_{\mathrm{L}}\right)$.

Réciproquement, supposons que pour tout $(N-2)$-plan $\mathrm{L}$ de $\mathbb{C}^{N}$ contenu dans l'hyperplan $H$ vérifiant $\mathrm{L} \cap\left|\mathrm{C}_{\mathrm{S}, 0}\right|=\{0\}$ le morphisme $f_{\mathrm{L}}: X_{\mathrm{L}} \rightarrow \mathrm{D}_{\mathrm{L}}$ a une résolution simultanée faible le long de $\sigma\left(\mathrm{D}_{\mathrm{L}}\right)$. Notons $x_{0}$ le point d'intersection de la transformée stricte, par l'éclatement $e_{\mathrm{L}}$, de $H \cap \mathrm{S}$ avec le diviseur exceptionnel de $e_{\mathrm{L}}$ et $d_{0}=f_{\mathrm{L}}\left(x_{0}\right)$. D'après le théorème 5.2 , pour tout $d \in \mathrm{D}_{\mathrm{L}}$, la fibre $f_{\mathrm{L}}^{-1}(d)$ est réduite et

$$
\mu\left(f_{\mathrm{L}}^{-1}(d), \sigma(d)\right)=\mu\left(f_{\mathrm{L}}^{-1}\left(d_{0}\right), x_{0}\right) .
$$

Or les fibres du morphisme $f_{\mathrm{L}}$ sont isomorphes aux sections hyperplanes de $\mathrm{S}$ dont elles sont les transformées strictes par $e_{\mathrm{L}}$. L'ensemble des hyperplans généraux de $\mathbb{C}^{N}$ étant un ouvert dense de l'ensemble des hyperplans de $\mathbb{C}^{N}$, il 
existe $d \in \mathrm{D}_{\mathrm{L}}$ tel que la fibre $f_{\mathrm{L}}^{-1}(d)$ soit définie par un hyperplan général $H^{\prime}$ de $\mathbb{C}^{N}$. On a alors

$$
\mu\left(f_{\mathrm{L}}^{-1}(d), \sigma(d)\right)=\mu\left(H^{\prime} \cap \mathrm{S}, 0\right) .
$$

Or d'après le théorème 4.2 , on a $\mu\left(H^{\prime} \cap \mathrm{S}, 0\right)=\mu_{\text {min }}$. Par conséquent,

$$
\mu(H \cap \mathrm{S}, 0)=\mu\left(f_{\mathrm{L}}^{-1}\left(d_{0}\right), x_{0}\right)=\mu\left(f_{\mathrm{L}}^{-1}(d), \sigma(d)\right)=\mu\left(H^{\prime} \cap \mathrm{S}, 0\right)=\mu_{m i n} .
$$

D'après le théorème 4.2, l'hyperplan $H$ est général.

Ce théorème nous donne une caractérisation des limites d'hyperplans tangents en termes d'existence d'une résolution simultanée faible de familles de sections hyperplanes. Rappelons qu'une tangente exceptionnelle de $\mathrm{S}$ en 0 est une génératrice du cône tangent telle que tout hyperplan la contenant est une limite d'hyperplan tangent à $\mathrm{S}$ en 0 . Cependant les tangentes exceptionnelles de $\mathrm{S}$ en 0 n'apparaissent pas clairement dans un éclatement de "type $e_{\mathrm{L}}$ ". Par contre, toute génératrice du cône tangent à $\mathrm{S}$ en 0 correspond à un point du diviseur exceptionnel de l'éclatement de l'origine dans $\mathrm{S}$.

5.5. Notons $e: \mathrm{S}^{\prime} \rightarrow \mathrm{S}$ l'éclatement de l'origine dans $\mathrm{S}$. La surface $\mathrm{S}^{\prime}$ obtenue est plongée dans $\mathrm{S} \times \mathbb{P}^{N-1}$. La courbe exceptionnelle dans $\mathrm{S}^{\prime}$ est la courbe projective $\operatorname{proj}_{\mathrm{S}, 0}$. Si $\mathrm{L}$ est un $(N-2)$-plan vérifiant $\mathrm{L} \cap\left|\mathrm{C}_{\mathrm{S}, 0}\right|=\{0\}$ alors, l'identité sur $\mathrm{S}$ et la projection : $\mathbb{P}^{N-1}-\operatorname{proj} \mathrm{L} \rightarrow \mathbb{P}^{1}$ induisent un morphisme $p_{\mathrm{L}}^{\prime}: \mathrm{S}^{\prime} \rightarrow \mathrm{S}_{\mathrm{L}}^{\prime}$. D'après [19] (II. $\S 7$ proposition 6), le morphisme $p_{\mathrm{L}}^{\prime}$ est un morphisme fini.

Théorème 5.6. Soit $l_{0}$ une génératrice du cône $\left|\mathrm{C}_{\mathrm{S}, 0}\right|$, notons $y_{0}$ le point qui lui correspond dans proj $\left|\mathrm{C}_{\mathrm{S}, 0}\right|$. La génératrice $l_{0}$ est une tangente exceptionnelle de la surface $\mathrm{S}$ en 0 si et seulement si, pour tout $(N-2)$-plan $\mathrm{L}$ vérifiant $\mathrm{L} \cap\left|\mathrm{C}_{\mathrm{S}, 0}\right|=\{0\}$, le morphisme $f_{\mathrm{L}}: X_{\mathrm{L}} \rightarrow \mathrm{D}_{\mathrm{L}}$ construit autour de l'image $p_{\mathrm{L}}^{\prime}\left(y_{0}\right)$ comme dans 5.3, n'a pas de résolution simultanée faible le long du diviseur exceptionnel.

Démonstration: Soit $l_{0}$ une tangente exceptionnelle de $\mathrm{S}$ en 0 et soit $\mathrm{L}$ un $(N-2)$-plan de $\mathbb{C}^{N}$ vérifiant $\mathrm{L} \cap\left|\mathrm{C}_{\mathrm{S}, 0}\right|=\{0\}$. Notons $y_{0}$ le point de $\operatorname{proj}\left|\mathrm{C}_{\mathrm{S}, 0}\right|$ correspondant à la génératrice $l_{0}$ et $x_{0}$ son image par le morphisme $p_{\mathrm{L}}^{\prime}$ construit comme dans 5.5. D'après la définition 2.9, l'hyperplan $H$ de $\mathbb{C}^{N}$ engendré par le $(N-2)$-plan L et la droite $l_{0}$ est une limite d'hyperplans tangents à la surface $\mathrm{S}$ en 0 et donc d'après le théorème 4.2 , la courbe $H \cap \mathrm{S}$ est soit non-réduite, soit réduite et $\mu(H \cap \mathrm{S}, 0)>\mu_{\min }$, où $\mu_{\min }$ désigne la valeur minimale des nombres de Milnor à l'origine des sections hyperplanes réduites. Cette section hyperplane est isomorphe à la fibre $f_{\mathrm{L}}^{-1}\left(d_{0}\right)$ du morphisme $f_{\mathrm{L}}: X_{\mathrm{L}} \rightarrow \mathrm{D}_{\mathrm{L}}$ (construit comme dans 5.3), où $d_{0}=f_{\mathrm{L}}\left(x_{0}\right)$.

Si la courbe $f_{\mathrm{L}}^{-1}\left(d_{0}\right)$ n'est pas réduite, alors le morphisme $f_{\mathrm{L}}$ n'a pas de résolution simultanée faible. 
Si la courbe $f_{\mathrm{L}}^{-1}\left(d_{0}\right)$ est réduite alors on a

$$
\mu\left(f_{\mathrm{L}}^{-1}\left(d_{0}\right), x_{0}\right)>\mu_{\min } .
$$

Par ailleurs, en choisissant un représentant $X_{\mathrm{L}}$ du germe de surface $\left(\mathrm{S}_{\mathrm{L}}^{\prime}, x_{0}\right)$ assez petit, on peut supposer que toutes les autres fibres du morphisme $f_{\mathrm{L}}$ sont les transformées strictes par $e_{\mathrm{L}}$ de sections hyperplanes définies par des hyperplans généraux de $\mathbb{C}^{N}$; donc pour tout $d \in \mathrm{D}_{\mathrm{L}}$ différent de $d_{0}$,

$$
\mu\left(f_{\mathrm{L}}^{-1}(d), \sigma(d)\right)=\mu_{\min }<\mu\left(f_{\mathrm{L}}^{-1}\left(d_{0}\right), x_{0}\right) .
$$

Par conséquent, d'après le théorème 5.2 , le morphisme $f_{\mathrm{L}}$ n'a pas de résolution simultanée faible le long du diviseur exceptionnel dans $X_{\mathrm{L}}$.

Réciproquement, supposons que pour tout $(N-2)$-plan $\mathrm{L}$ de $\mathbb{C}^{N}$ vérifiant $\mathrm{L} \cap\left|\mathrm{C}_{\mathrm{S}, 0}\right|=\{0\}$ le morphisme $f_{\mathrm{L}}: X_{\mathrm{L}} \rightarrow \mathrm{D}_{\mathrm{L}}$ construit comme dans 5.3 autour du point $x_{0}=p_{\mathrm{L}}^{\prime}\left(y_{0}\right)$ n'admet pas de résolution simultanée faible le long du diviseur exceptionnel dans $X_{\mathrm{L}}$.

Soit $H$ un hyperplan de $\mathbb{C}^{N}$ contenant la génératrice $l_{0}$. Si l'hyperplan $H$ contient une composante irréductible de $\left|\mathrm{C}_{\mathrm{S}, 0}\right|$ alors c'est une limite d'hyperplans tangents. Sinon, l'hyperplan $H$ contient un $(N-2)$-plan L de $\mathbb{C}^{N}$ vérifiant $\mathrm{L} \cap\left|\mathrm{C}_{\mathrm{S}, 0}\right|=\{0\}$. Puisque le morphisme $f_{\mathrm{L}}: X_{\mathrm{L}} \rightarrow \mathrm{D}_{\mathrm{L}}$ induit par L n'a pas de résolution simultanée faible le long du diviseur exceptionnel dans $X_{\mathrm{L}}$ alors d'après le théorème 5.4, l'hyperplan $H$ n'est pas général. Par conséquent, tout hyperplan de $\mathbb{C}^{N}$ contenant la génératrice $l_{0}$ est une limite d'hyperplans tangents à la surface $\mathrm{S}$ en 0 . D'après la définition 2.9 , la génératrice $l_{0}$ est une tangente exceptionnelle de la surface $\mathrm{S}$ en 0 . Ce qui achève la preuve du théorème 5.6.

Remarque 5.7. Notons $f$ et $g$ deux fonctions holomorphes de $\mathcal{O}_{\mathrm{S}, 0}$ qui définissent la projection $\pi_{\mathrm{L}}: \mathrm{S} \rightarrow U$. D'après [24] (I. §5.2), la projection $\pi_{\mathrm{L}}$ est générique si et seulement si l'idéal maximal $\mathfrak{m}$ de $\mathcal{O}_{\mathrm{S}, 0}$ est entier sur l'idéal $(f, g) \mathcal{O}_{\mathrm{S}, 0}$. Par conséquent, lorsque la projection $\pi_{\mathrm{L}}$ est générique les deux idéaux $(f, g)$ et $\mathfrak{m}$ de $\mathcal{O}_{\mathrm{S}, 0}$ ont màme clôture intégrale (en l'occurence $\mathfrak{m}$ ); d'après [24] (page 330), ces deux idéaux ont màme éclatement normalisé.

Nous avons alors le diagramme commutatif suivant:

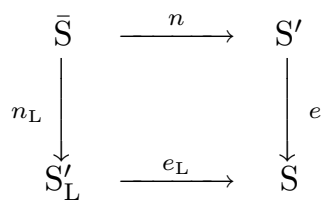

où les morphismes $n$ et $n_{\mathrm{L}}$ sont respectivement les normalisations des surfaces $\mathrm{S}^{\prime}$ et $\mathrm{S}_{\mathrm{L}}^{\prime}$.

Nous pouvons maintenant déterminer explicitement les tangentes exceptionnelles d'une surface normale: 
Théorème 5.8. Soient $l_{0}$ une génératrice du cône $\left|\mathrm{C}_{\mathrm{S}, 0}\right|$ et $y_{0}$ le point de $\operatorname{proj}\left|\mathrm{C}_{\mathrm{S}, 0}\right|$ qui lui correspond. La génératrice $l_{0}$ est une tangente exceptionnelle de la surface $\mathrm{S}$ en 0 si et seulement si $y_{0}$ est un point singulier d'une branche de la courbe proj $\left|\mathrm{C}_{\mathrm{S}, 0}\right|$ ou l'image par la normalisation $n$ d'un point singulier de la surface $\overline{\mathrm{S}}$ ou d'un point singulier du diviseur exceptionnel dans $\overline{\mathrm{S}}$ ou une valeur critique de la restriction de la normalisation au diviseur exceptionnel de $\overline{\mathrm{S}}$.

Nous appellerons point spécial un point de la courbe $\operatorname{proj}\left|\mathrm{C}_{\mathrm{S}, 0}\right|$ parmi ceux énumérés dans ce théorème.

Ce théorème améliore le résultat annoncé dans [23] (théorème 8) et démontré dans [22] (théorème 4.17). Nous remercions le professeur D. T. Là qui a attiré notre attention sur cette amélioration.

Remarque 5.9. Dans [9] (définition 4), G. González-Sprinberg et M. LejeuneJalabert appellent section hyperplane générale d'une surface $\mathrm{S}$ (non-nécessairement normale), une section de $\mathrm{S}$ définie par un hyperplan $H$ dont le projectivisé $\operatorname{proj} H$ est transverse à la courbe $\operatorname{proj}\left|\mathrm{C}_{\mathrm{S}, 0}\right|$ en évitant: la transformée stricte par l'éclatement $e \mathrm{du}$ lieu singulier de $\mathrm{S}$, les points singuliers et les points spéciaux de $\operatorname{proj}\left|\mathrm{C}_{\mathrm{S}, 0}\right|$. Le théorème ci-dessus nous permet de comparer les sections hyperplanes générales dans le sens de [9] aux sections hyperplanes définies par des hyperplans généraux dans le sens de ce travail; voir la proposition 5.12.

Démonstration: Montrons d'abord qu'un point spécial correspond à une tangente exceptionnelle de $\mathrm{S}$ en 0 :

Soit $y_{0}$ un point de $\operatorname{proj}\left|\mathrm{C}_{\mathrm{S}, 0}\right|$ et $\mathrm{L}$ un $(N-2)$-plan de $\mathbb{C}^{N}$ vérifiant $\mathrm{L} \cap$ $\left|\mathrm{C}_{\mathrm{S}, 0}\right|=\{0\}$. Notons $p_{\mathrm{L}}^{\prime}: \mathrm{S}^{\prime} \rightarrow \mathrm{S}_{\mathrm{L}}^{\prime}$ le morphisme induit par L (comme dans 5.5) et $x_{0}=p_{\mathrm{L}}^{\prime}\left(y_{0}\right)$. Soit $f_{\mathrm{L}}: X_{\mathrm{L}} \rightarrow \mathrm{D}_{\mathrm{L}}$ le morphisme construit autour de $x_{0}$ comme dans 5.3.

1 er cas: Le point $y_{0}$ est l'image par la normalisation $n$ d'un point singulier de $\overline{\mathrm{S}}$. Dans ce cas, par commutativité du diagramme (5.2), la surface normalisée de $X_{\mathrm{L}}$ est singulière au dessus de $x_{0}$; par conséquent, le morphisme $f_{\mathrm{L}}$ n'a pas de normalisation en famille, il n'a donc pas de résolution simultanée faible. D'après le théorème 5.6 , la génératrice $l_{0}$ correspondant à $y_{0}$ est une tangente exceptionnelle de $\mathrm{S}$ en 0.

2 ème cas: $y_{0}$ est l'image par la normalisation $n$ d'un point singulier du diviseur exceptionnel de $\overline{\mathrm{S}}$. Le point $x_{0}=p_{\mathrm{L}}^{\prime}\left(y_{0}\right)$ est alors image par $n_{\mathrm{L}}$ d'un point singulier du diviseur exceptionnel de $\overline{\mathrm{S}}$. Il est donc impossible d'avoir $\left|n_{\mathrm{L}}^{-1}\left(\sigma\left(\mathrm{D}_{\mathrm{L}}\right)\right)\right| \stackrel{\simeq}{=} \mathrm{D}_{\mathrm{L}} \times\left|n_{\mathrm{L}}^{-1}\left(x_{0}\right)\right|$. Le morphisme $f_{\mathrm{L}}$ n'a donc pas de résolution simultanée faible le long de $\sigma\left(\mathrm{D}_{\mathrm{L}}\right)$. D'après le théorème 5.6 , la génératrice $l_{0}$ est une tangente exceptionnelle de $\mathrm{S}$ en 0.

3 ème cas: Le point $y_{0}$ est une valeur critique de la restriction de la normalisation $n$ au diviseur exceptionnel dans $\overline{\mathrm{S}}$. Si le morphisme $f_{\mathrm{L}}$ avait une résolution simultanée faible le long du diviseur exceptionnel dans $X_{\mathrm{L}}$ alors on aurait d'après 
5.1:

$$
\left|n_{\mathrm{L}}^{-1}\left(\sigma\left(\mathrm{D}_{\mathrm{L}}\right)\right)\right| \stackrel{\sim}{=} \mathrm{D}_{\mathrm{L}} \times\left|n_{\mathrm{L}}^{-1}\left(x_{0}\right)\right| .
$$

Or d'après la commutativité du diagramme (5.2), on a $n_{\mathrm{L}}=p_{\mathrm{L}}^{\prime}$ on, par conséquent, si on note $Y$ un voisinage de $y_{0}$ dans $\mathrm{S}^{\prime}$ au dessus de $X_{\mathrm{L}}$, on aurait:

$$
\left|n^{-1}\left(\operatorname{proj}\left|\mathrm{C}_{\mathrm{S}, 0}\right| \cap Y\right)\right| \simeq \mathrm{D}_{\mathrm{L}} \times\left|n^{-1}\left(y_{0}\right)\right|,
$$

ce qui est impossible quand $y_{0}$ est une valeur critique de la restriction de $n$ au diviseur exceptionnel de $\overline{\mathrm{S}}$. Le morphisme $f_{\mathrm{L}}$ n'a donc pas de résolution simultanée faible. D'après le théorème 5.6, la génératrice $l_{0}$ est une tangente exceptionnelle de $\mathrm{S}$ en 0 .

4 ème cas: Le point $y_{0}$ est un point singulier d'une branche de $\operatorname{proj}\left|\mathrm{C}_{\mathrm{S}, 0}\right|$. Il existe un point $s_{0} \in \overline{\mathrm{S}}$ tel que $n\left(s_{0}\right)=y_{0}$ et l'image par $n$ du diviseur exceptionnel au voisinage de $s_{0}$ contienne la branche de $\operatorname{proj}\left|\mathrm{C}_{\mathrm{S}, 0}\right|$ singulière en $y_{0}$. Dans ce cas, au voisinage de $s_{0}$, la restriction de la normalisation $n_{\mathrm{L}}$ au diviseur exceptionnel se factorise par une courbe singulière. Par conséquent le point $x_{0}=p_{\mathrm{L}}^{\prime}\left(y_{0}\right)$ est une valeur critique de cette restriction. Le morphisme $f_{\mathrm{L}}$ n'a donc pas de résolution simultanée faible, et donc d'après le théorème 5.6 , la génératrice $l_{0}$ est une tangente exceptionnelle de $\mathrm{S}$ en 0 .

Réciproquement, soit $y_{0}$ un point de $\operatorname{proj}\left|\mathrm{C}_{\mathrm{S}, 0}\right|$ non-spécial, montrons que la génératrice $l_{0}$ de $\left|\mathrm{C}_{\mathrm{S}, 0}\right|$ qui lui correspond n'est pas une tangente exceptionnelle de $\mathrm{S}$ en 0 .

Choisissons un $(N-2)$-plan $\mathrm{L}$ de $\mathbb{C}^{N}$ verifiant $\mathrm{L} \cap\left|\mathrm{C}_{\mathrm{S}, 0}\right|=\{0\}$ et tel que l'hyperplan $H_{0}$ de $\mathbb{C}^{N}$ contenant $\mathrm{L}$ et $l_{0}$ vérifie les conditions suivantes:

$$
\left\{\begin{array}{l}
\text { la courbe } H_{0} \cap \mathrm{S} \text { est réduite } \\
\text { proj } H_{0} \text { ne contient pas de point spécial } \\
H_{0} \text { n'est pas tangent au cône }\left|\mathrm{C}_{\mathrm{S}, 0}\right| .
\end{array}\right.
$$

Soit $p_{\mathrm{L}}^{\prime}: \mathrm{S}^{\prime} \rightarrow \mathrm{S}_{\mathrm{L}}^{\prime}$ le morphisme induit par $\mathrm{L}$ comme dans 5.5. Posons $x_{0}=p_{\mathrm{L}}^{\prime}\left(y_{0}\right)$ et notons $f_{\mathrm{L}}: X_{\mathrm{L}} \rightarrow \mathrm{D}_{\mathrm{L}}$ le morphisme construit autour de $x_{0}$ comme dans 5.3. Posons $d_{0}=f_{\mathrm{L}}\left(x_{0}\right)$.

Nous allons montrer que le morphisme $f_{\mathrm{L}}$ a une résolution simultanée faible le long du diviseur exceptionnel dans $X_{\mathrm{L}}$.

Le point $d_{0}$ n'est pas une valeur critique de la restriction de $f_{\mathrm{L}} \circ n_{\mathrm{L}}$ au diviseur exceptionnel de $\overline{\mathrm{S}}$. En effet, Soit $z_{0}$ un point de $\overline{\mathrm{S}}$ au-dessus de $x_{0}$. Par commutativité du diagramme $(5.2), n\left(z_{0}\right)=y_{0}$ et puisque $y_{0}$ n'est pas un point spécial, $z_{0}$ n'est pas un point singulier du diviseur exceptionnel réduit de $\overline{\mathrm{S}}$ et son image par la normalisation est un point non-singulier d'une branche de $\operatorname{proj} \mathrm{C}_{\mathrm{S}, 0}$. Par construction du morphisme $p_{\mathrm{L}}^{\prime}$ (voir 5.5), la restriction à cette branche de $\operatorname{proj} \mathrm{C}_{\mathrm{S}, 0}$ du morphisme $f_{\mathrm{L}} \circ p_{\mathrm{L}}^{\prime}$ est critique en $y_{0}$ si et seulement si l'hyperplan projectif $\operatorname{proj} H_{0}$ n'est pas transverse à cette branche dans $\mathbb{P}^{N-1}$; ce qui est exclu par le choix de $H_{0}$. Le point $z_{0}$ n'est pas alors un point critique du morphisme composé $f_{\mathrm{L}} \circ p_{\mathrm{L}}^{\prime} \circ n$ restreint au diviseur exceptionnel de $\overline{\mathrm{S}}$. Ainsi, 
le point $d_{0}$ n'est pas une valeur critique de la restriction de $f_{\mathrm{L}} \circ n_{\mathrm{L}}$ au diviseur exceptionnnel de $\overline{\mathrm{S}}$.

Il en résulte que la fibre $\left(f_{\mathrm{L}} \circ n_{\mathrm{L}}\right)^{-1}\left(d_{0}\right)$ est transverse au diviseur exceptionnel de $\overline{\mathrm{S}}$, de plus la section hyperplane $H_{0} \cap \mathrm{S}$ étant réduite la fibre $f_{\mathrm{L}}^{-1}\left(d_{0}\right)$ est une courbe réduite. Le morphisme $f_{\mathrm{L}}$ a donc une normalisation en famille au voisinage de $x_{0}$.

Montrons maintenant que le nombre de branches de la fibre $f_{\mathrm{L}}^{-1}\left(d_{0}\right)$ en $x_{0}$ est égal au nombre de branches de $f_{\mathrm{L}}^{-1}(d)$ en $\sigma(d)$ pour $d$ voisin de $d_{0}$ et où $\sigma$ est la section naturelle de $\mathbb{P}^{1}$ dans $\mathrm{S}_{\mathrm{L}}^{\prime}$. Posons $x=\sigma(d)$.

Puisque les fibres $\left(f_{\mathrm{L}} \circ n_{\mathrm{L}}\right)^{-1}\left(d_{0}\right)$ et $\left(f_{\mathrm{L}} \circ n_{\mathrm{L}}\right)^{-1}(d)$ sont non-singulières, le nombre de branches de $f_{\mathrm{L}}^{-1}\left(d_{0}\right)$ en $x_{0}$ et de $f_{\mathrm{L}}^{-1}(d)$ en $x$ est respectivement égal au cardinal de $n_{\mathrm{L}}^{-1}\left(x_{0}\right)$ et de $n_{\mathrm{L}}^{-1}(x)$.

Si $y_{i}$ est un point de $\operatorname{proj} \mathrm{C}_{\mathrm{S}, 0}$ au-dessus de $x_{0}$, et puisque l'hyperplan projectif $\operatorname{proj}_{0}$ est transverse à chacune des branches de $\operatorname{proj} \mathrm{C}_{\mathrm{S}, 0}$ en $y_{i}$ dans $\mathbb{P}^{N-1}$, le nombre de points $y_{i, j}$ de $\operatorname{proj} \mathrm{C}_{\mathrm{S}, 0}$ au-dessus de $x$ voisins de $y_{i}$ est égal au nombre de branches de la courbe $\operatorname{proj}\left|\mathrm{C}_{\mathrm{S}, 0}\right|$ en $y_{i}$. D'autre part, en vertu des conditions (5.3), le cardinal de chaque fibre $n^{-1}\left(y_{i}\right)$ est égal à la somme des cardinaux des fibres $n^{-1}\left(y_{i, j}\right)$ pour tous les points $y_{i, j}$ au-dessus de $x$ voisins de $y_{i}$. Par commutativité du diagramme (5.2), le cardinal de la fibre $n_{\mathrm{L}}^{-1}\left(x_{0}\right)$ est donc égal à celui de la fibre $n_{\mathrm{L}}^{-1}(x)$ et par conséquent les courbes $f_{\mathrm{L}}^{-1}\left(d_{0}\right)$ et $f_{\mathrm{L}}^{-1}(d)$ ont le màme nombre de branches respectivement en $x_{0}$ et en $x$.

D'après le théorème 5.2 , le morphisme $f_{\mathrm{L}}$ a une résolution simultanée faible le long du diviseur exceptionnel et donc d'après le théorème 5.6, la génératrice $l_{0}$ du cône $\mathrm{C}_{\mathrm{S}, 0}$ n'est pas une tangente exceptionnelle de la surface $\mathrm{S}$ en 0 . Ce qui achève la preuve du théorème 5.8 .

Remarque 5.10. D'après le théorème 5.8 , un point de croisement de composantes irréductibles non-singulières de la courbe $\operatorname{proj}_{\mathrm{S}, 0}$ qui n'est pas un point spécial ne correspond pas à une tangente exceptionnelle de la surface $\mathrm{S}$ en 0 . Dans le cas où la surface $\mathrm{S}$ est une hypersurface à singularités isolées, de tels points n'existent pas puisque J. P. G. Henry et D. T. Là ont prouvé dans [14], que tous les points singuliers de $\operatorname{proj}\left|\mathrm{C}_{\mathrm{S}, 0}\right|$ correspondent à des tangentes exceptionnelles. Il serait intéressant de savoir si de tels points existent dans le cas général des surfaces normales.

Dans le cas où la surface $S^{\prime}$ obtenue par éclatement de l'origine de $\mathrm{S}$ est ellemàme une surface normale (c'est le cas en particulier des surfaces à singularités rationnelles (cf. [29])), le résultat du théorème 5.8 s'exprime plus simplement:

Corollaire 5.11. Dans le cas où l'éclatement de l'origine d'une surface normale $\mathrm{S}$ est une surface normale, une génératrice $l_{0}$ du cône $\mathrm{C}_{\mathrm{S}, 0}$ est une tangente exceptionnelle de $\mathrm{S}$ en 0 si et seulement si le point $y_{0}$ de proj $\left|\mathrm{C}_{\mathrm{S}, 0}\right|$ lui correspondant est un point singulier de la surface éclatée $\mathrm{S}^{\prime}$ ou un point singulier de 
la courbe proj $\left|\mathrm{C}_{\mathrm{S}, 0}\right|$.

Ce corollaire découle du fait que dans le diagramme (5.2), les surfaces $\mathrm{S}^{\prime}$ et $\overline{\mathrm{S}}$ sont égales. On applique alors le théorème 5.8 .

En vertu des théorèmes $2.8,5.8$, du corollaire 5.11 et de la remarque 5.9 , on a:

Proposition 5.12. Pour une surface S normale en 0, une section hyperplane générale dans le sens de [9] est définie par un hyperplan qui n'est pas limite d'hyperplans tangents à la surface $\mathrm{S}$ en 0 .

Dans le cas où l'éclaté de $\mathrm{S}$ en 0 est une surface normale, une section hyperplane est générale dans le sens de [9] si et seulement si elle est définie par un hyperplan qui n'est pas une limite d'hyperplans tangents à la surface $\mathrm{S}$ en 0.

\section{Courbes polaires}

La notion de variétés polaires introduite et étudiée par D.T. Là et B. Teissier (cf. [15]), constitue une classe d'objets géométriques liés à l'étude géométrique et topologique des singularités des espaces analytiques complexes. Dans le cas des germes de surfaces complexes, cette notion se réduit à celle des courbes polaires qui se trouvent àtre intimement liée aux tangentes exceptionnelles en des points singuliers.

Un $(N-2)$-plan linéaire $\mathrm{L}$ de $\mathbb{C}^{N}$ induit un morphisme $\pi_{\mathrm{L}}$ de la surface $\mathrm{S}$ sur un ouvert $U$ de $\mathbb{C}^{2}$. D'après [15] ( $\left.\$ 2\right)$, on définit les courbes polaires associées au germe de surface $(\mathrm{S}, 0)$ comme suit:

Définition 6.1. Pour un $(N-2)$-plan générique L de $\mathbb{C}^{N}$, l'adhérence dans $\mathrm{S}$ du lieu critique de la restriction de $\pi_{\mathrm{L}}$ au lieu non-singulier de $\mathrm{S}$ est un espace analytique reduit de dimension 1 ou vide qu'on appelle courbe polaire (ou première variété polaire) associée au germe de surface $(\mathrm{S}, 0)$ et au $(N-2)$-plan $\mathrm{L}$ et qu'on note $\mathrm{P}_{1}((\mathrm{~S}, 0), \mathrm{L})$.

Dans le cas d'une surface normale, et à moins que la surface ne soit nonsingulière, les courbes polaires ne sont pas vides et coincident avec le lieu critique des projections linéaires génériques.

On définit dans [21] (III. définition 1.1) les points fixes des courbes polaires comme suit:

Définition 6.2. Soit $\rho: X \rightarrow \mathrm{S}$ une modification de la surface $\mathrm{S}$ au-dessus de 0 . On dira qu'un point $\eta$ de la fibre exceptionnelle de $\rho$ est un point fixe par $\rho$ du système linéaire des courbes polaires ou encore un point base pour $\rho$ s'il existe un ouvert dense $\Omega$ de la Grassmannienne des $(N-2)$-plans de $\mathbb{C}^{N}$, tel que pour tout $\mathrm{L} \in \Omega$, la transformée stricte par $\rho$ de la courbe polaire $\mathrm{P}_{1}((\mathrm{~S}, 0), \mathrm{L})$ 
contienne le point $\eta$.

On commencera par étudier les points fixes du système linéaire des courbes polaires par l'éclatement de l'origine d'une surface normale.

Un résultat de D.T. Là et B. Teissier, dans [16] (proposition 2.2.1) s'interprète dans le cas des surfaces normales comme suit:

Proposition 6.3. Un point de proj $\left|\mathrm{C}_{\mathrm{S}, 0}\right|$ est un point base (ou encore un point fixe des courbes polaires) pour l'éclatement de l'origine dans $\mathrm{S}$ si et seulement si la génératrice du cône tangent qui lui correspond est une tangente exceptionnelle de la surface en 0 .

Dans le cas des surfaces à singularités rationnelles, M. Spivakovsky démontre dans [21] (III. corollaire 3.11) le résultat suivant:

Proposition 6.4. Soit S une surface normale ayant une singularité rationnelle en 0 et soit $e: \mathrm{S}^{\prime} \rightarrow \mathrm{S}$ l'éclatement de l'origine. Un point non-singulier de la surface $\mathrm{S}^{\prime}$ est un point base pour l'éclatement si et seulement s'il est un point singulier du diviseur exceptionnel réduit.

Et dans [21] (III. Remarque 3.12) il pose la question suivante:

Question 6.5. Soit $S^{\prime}$ la surface obtenue par l'éclatement d'une singularité rationnelle de surface. Un point singulier de la surface $S^{\prime}$ est-il un point base pour l'éclatement?

Il donne une réponse positive à cette question dans le cas d'une surface à singularité minimale dans [21] (III. §5).

Le corollaire 5.11 énoncé dans la section précédente donne une réponse positive à la question 6.5 ainsi qu'une preuve de la proposition 6.4.

En appliquant les résultats obtenus dans la section précédente sur les tangentes exceptionnelles d'une surface normale, nous obtenons une réponse positive à la question 6.5 dans le cas général d'une surface normale:

Théorème 6.6. Soit $S$ une surface normale à singularité en 0 . Notons e : $\mathrm{S}^{\prime} \rightarrow \mathrm{S}$ l'éclatement de l'origine dans $\mathrm{S}$ et $n: \overline{\mathrm{S}} \rightarrow \mathrm{S}^{\prime}$ la normalisation de $\mathrm{S}^{\prime}$. L'image par $n$ d'un point singulier de $\overline{\mathrm{S}}$ est un point base pour l'éclatement $e$.

Ce théorème est une conséquence immédiate du théorème 5.8 et de la proposition 6.3.

Nous allons maintenant redonner une formulation à ce résultat en termes de contraction des composantes de Tyurina.

Donnons d'abord une définition qui généralise la notion de composantes de Tyurina à certaines résolutions d'une surface normale. 
Considérons une résolution $\pi: X \rightarrow \mathrm{S}$ de la surface $\mathrm{S}$. Si l'on note $\mathfrak{m}$ l'idéal maximal de l'anneau $\mathcal{O}_{\mathrm{S}, 0}$, alors, d'après la propriété universelle de l'éclatement et de la normalisation (cf. [2] page 25), le faisceau $\mathfrak{m} \mathcal{O}_{X}$ est inversible si et seulement si la résoltion $\pi: X \rightarrow \mathrm{S}$ se factorise par l'éclatement normalisé de l'idéal maximal $\mathfrak{m}$.

Considérons alors une résolution $\pi: X \rightarrow \mathrm{S}$ telle que le faisceau $\mathfrak{m} \mathcal{O}_{X}$ soit inversible. Notons $e: \mathrm{S}^{\prime} \rightarrow \mathrm{S}$ l'éclatement de l'origine dans $\mathrm{S}$ et $n: \overline{\mathrm{S}} \rightarrow \mathrm{S}^{\prime}$ sa normalisation, et notons $\bar{\pi}: X \rightarrow \overline{\mathrm{S}}$ le morphisme de factorisation: $\pi=e \circ n \circ \bar{\pi}$.

Notons $\left|\pi^{-1}(0)\right|=\bigcup_{i=1}^{n} \mathrm{E}_{i}$ la décomposition de la fibre exceptionnelle de la résolution en composantes irrédutibles. Si $f$ désigne une fonction holomorphe contenue dans l'idéal maximal $\mathfrak{m}$, on désignera par $\mathrm{D}_{f}$ la partie du diviseur $\pi^{*} f$ qui est à support sur la fibre exceptionnelle. Il existe alors des entiers naturels $m_{1, f}, \cdots, m_{n, f}$ tels que $\mathrm{D}_{f}=\sum_{i=1}^{n} m_{i, f} \mathrm{E}_{i}$. On définit le cycle maximal de la résolution $\pi$ (cf. [31] II. §4), comme étant le cycle

$$
\mathcal{M}_{X}=i n f_{f \in m} \mathrm{D}_{f}
$$

où l'on définit l'ordre partiel sur les cycles à support sur la fibre exceptionnelle en comparant les coefficients un par un.

On posera

$$
\mathcal{M}_{X}=\sum_{i=1}^{n} m_{i} \mathrm{E}_{i} .
$$

C'est un cycle positif qui vérifie $\left(\mathcal{M}_{X} \cdot \mathrm{E}_{i}\right) \leq 0$ pour toute $i \in\{1 \cdots n\}$ (cf. [31] II. $\S 4)$.

Le théorème suivant est prouvé dans [8] §2:

Théorème 6.7. Une composante irréductible $\mathrm{E}_{i}$ de la fibre exceptionnelle de la résolution $\pi$ se contracte par $\bar{\pi}$ sur un point du diviseur exceptionnel dans $\overline{\mathrm{S}}$ si et seulement si $\left(\mathcal{M}_{X} \cdot \mathrm{E}_{i}\right)=0$.

De màme l'image par $\bar{\pi}$ d'une composante irréductible $\mathrm{E}_{i}$ est une composante irréductible du diviseur exceptionnel dans $\overline{\mathrm{S}}$ si et seulement si $\left(\mathcal{M}_{X} \cdot \mathrm{E}_{i}\right)<0$.

Une démonstration de ce résultat est également faite dans [22] (théorème 3.5).

Notons $\Delta_{1}, \cdots, \Delta_{k}$ les composantes connexes de l'adhérence dans $\left|\pi^{-1}(0)\right|$ de l'ensemble $\left|\pi^{-1}(0)\right|-\underset{\left(\mathcal{M}_{X} \cdot \mathrm{E}_{i}\right)<0}{\bigcup} \mathrm{E}_{i}$.

Définition 6.8. On appellera les composantes connexes $\Delta_{i}$ définies ci-dessus, les composantes de Tyurina-Spivakovsky de la résolution $\pi: X \rightarrow \mathrm{S}$.

Comme l'a remarqué M. Spivakovsky, dans [21] (III. Lemme 7.1), les com- 
posantes $\Delta_{i}$ définies ci-dessus généralisent la notion de composantes de Tyurina d'une résolution d'une surface à singularités rationnelles (cf. [29]) au cas des résolutions de surfaces normales qui se factorisent par l'éclatement normalisé des points singuliers.

Nous pouvons maintenant reformuler le théorème 6.6 en termes de contractions des composantes de Tyurina-Spivakovsky:

Théorème 6.9. $\quad$ Si $\pi: X \rightarrow \mathrm{S}$ est une désingularisation de la surface $\mathrm{S}$ obtenue par composition de l'éclatement $e: \mathrm{S}^{\prime} \rightarrow \mathrm{S}$ de l'idéal maximal $\mathfrak{m} \mathcal{O}_{\mathrm{S}, 0}$, de la normalisation $n: \overline{\mathrm{S}} \rightarrow \mathrm{S}^{\prime}$ de $\mathrm{S}^{\prime}$ et de la désingularisation minimale $\bar{\pi}: X \rightarrow \overline{\mathrm{S}}(\pi=e \circ n \circ \bar{\pi})$, alors les composantes de Tyurina-Spivakovsky de la désingularisation $\pi$ se contractent sur des points bases des courbes polaires de $\mathrm{S}$ en 0 pour l'éclatement de l'idéal maximal de $\mathcal{O}_{\mathrm{S}, 0}$.

Démonstration: D'après la définition 6.8 et le théorème 6.7 , les composantes de Tyurina-Spivakovsky de la désingularisation $\pi$ se contractent par le morphisme $\bar{\pi}$ sur des points de la surface $\overline{\mathrm{S}}$. La résolution $\bar{\pi}$ de $\overline{\mathrm{S}}$ étant minimale, chaque composante de Tyurina-Spivakovsky de $\pi$ se contracte par $\bar{\pi}$ sur un point singulier de la surface $\overline{\mathrm{S}}$. D'après le théorème 5.8 et la proposition 6.3 , les composantes de Tyurina-Spivakovsky de $\pi$ se contractent donc par le morphisme $n \circ \bar{\pi}$ sur des points bases des courbes polaires de $\mathrm{S}$ en 0 par l'éclatement de l'idéal maximal de $\mathcal{O}_{\mathrm{S}, 0}$.

On peut également s'intéresser à l'étude de l'existence de points fixes du système linéaire des courbes polaires dans une désingularisation d'une surface normale. Ce problème est relié à l'étude des résolutions des surfaces par des suites de modifications de Nash normalisées.

Dans [21] (III. théorème 1.2), M. Spivakovsky démontre:

Proposition 6.10. Le système linéaire des courbes polaires de S n'a pas de point fixe dans la résolution $\pi: X \rightarrow \mathrm{S}$ si et seulement si la résolution $\pi$ se factorise par la modification de Nash normalisée de la surface $\mathrm{S}$.

La détermination explicite de la résolution d'une singularité de surface normale par des suites de modifications de Nash normalisées à partir d'une désingularisation donnée, passe donc par la détermination des points fixes du système linéaire des courbes polaires dans cette désingularisation.

Considérons une désingularisation $\pi: X \rightarrow \mathrm{S}$ pour laquelle le faisceau $\mathfrak{m} \mathcal{O}_{X}$ est inversible et qui soit minimale pour cette condition et notons $\sigma: X^{\prime} \rightarrow X$ la suite minimale d'éclatements de points pour laquelle le système linéaire des courbes polaires n'a pas de points fixes dans $X^{\prime}$. D'après la proposition 6.10 , la surface $X^{\prime}$ domine la modification de Nash normalisée $\tilde{\text { S }}$.

Nous obtenons alors le diagramme suivant, utilisé dans [21] (III. 2) et [6] (§2): 


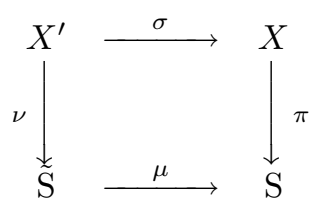

La minimalité des morphismes $\sigma$ et $\pi$ fait que les composantes de TyrinaSpivakovsky de la résolution $\pi \circ \sigma: X^{\prime} \rightarrow \mathrm{S}$ se contractent exactement sur les points fixes du système linéaire des courbes polaires dans l'éclatement de l'origine dans S, qui sont les tangentes exceptionnelles de la surface normale $\mathrm{S}$ en 0 .

Dans le cas où les singularités de la surface $S$ sont des points doubles rationnels ou des singularités minimales, G. González-Sprinberg dans [6] (§5) pour le premier cas et M. Spivakovsky dans [21] (III. §5) pour le second, déterminent les points fixes du système linéaire des courbes polaires dans la résolution minimale de la surface. Dans le cas général, il n'y a pas encore à notre connaissance de résultat sur la détermination des points fixes du système linéaire des courbes polaires dans une résolution des singularités.

\section{References}

[1] R. O. Buchweitz, G. M. Greuel, The Milnor number and deformations of complex curve singularities, Invent. Math. 583 (1980), 241-281.

[2] W. Barth, C. Peters, A. Van de Ven, Compact complex surfaces, Springer-Verlag, 1984.

[3] D. Eisenbud, Commutative algebra with a view toward algebraic geometry, G. T. of M. (150), Springer-Verlag, 1995.

[4] W. Fulton, Algebraic curves, W. A. Benjamin, 1969.

[5] G. González-Sprinberg, L'obstruction locale d'Euler et le théorème de MacPherson, Sem. E.N.S. 1978/1979, Astérisque 82-83 (1981), 7-32.

[6] G. González-Sprinberg, Résolution de Nash des points doubles rationnels, Ann. Inst. Fourier, Grenoble 322 (1982), 111-178.

[7] P. Griffiths, J. Harris, Principles of algebraic geometry, Wiley and Sons, 1978.

[8] G. González-Sprinberg, M. Lejeune-Jalabert, Courbes lisses, cycle maximal et points infiniment voisins des singularités de surfaces; Prépublication de l'institut Fourrier, Grenoble $n^{o}$ 196, 1992.

[9] G. González-Sprinberg, M. Lejeune-Jalabert, Courbes lisses sur les singularités de surfaces. C. R. Acad. Sci. Paris Sér. I Math. 318 no. 7 (1994), 653-656.

[10] M. Giusti, Classification des singularités isolées simples d'intersections complètes, Proc. of symposia in pure mathematics, Vol. 40 (1983) part 1.

[11] M. Giusti, Sur les singularités isolées d'intersections complètes quasi-homogènes, Ann. Inst. Fourier, Grenoble 273 (1977), 163-192.

[12] H. Hamm, Lokale topologische Eigenschaften komplexer Räume, Math. Ann. 191 (1971), $235-252$

[13] D. T. Là, Limites d'espaces tangents sur les surfaces. Nova Acta Leopoldina NF 52 Nr.240 (1981), 119-137.

[14] D. T. Lê, J. P. G. Henry, Limites d'espaces tangents, Sém. Norguet, Springer Lect. notes in Math. 482.

[15] D. T. Lê, B. Teissier, Variétés polaires locales et classes de Chern des variétés singulières, Annals of Math. 114 (1981), 457-491. 
[16] D. T. Lê, B. Teissier, Limites d'espaces tangents en géométrie anlytique, Comment. Math. Helvetici 63 (1988), 540-578.

[17] D. T. Lê, B. Teissier, Sur la géométrie des surfaces complexes I. Tangentes exceptionnelles. Amer. J. of Math. 101 (1979), 420-452.

[18] J. Milnor, Singular points of complex hypersurfaces, Ann. of Math. Studies 61, Princeton university press, 1968.

[19] D. Mumford, The red book for varieties and schemes, L.N.M. 1358, Springer-Verlag 1988.

[20] R. Narasimhan, Introduction to the theory of analytic spaces, L. N. 25, 1966, Springer-Verlag.

[21] M. Spivakovsky, Sandwiched singularities and desingularisation of surfaces by normalized Nash transformations, Annals of math. 131 (1990), 411-491.

[22] J. Snoussi, Limites d'espaces tangents à une surface normale, thèse de doctorat, Université de Provence - Marseille, 1998.

[23] J. Snoussi, Limites d'espaces tangents à une surface normale, C. R. Acad. Sci. Paris, T. 327 Série I (1998), 369-372.

[24] B. Teissier, Variétés polaires 2: Multiplicités polaires, sections planes, et conditions de Whitney. Actes de la conférence de géométrie algébrique à la Rabida, 1981, Springer Lecture Notes 961, pp. 314-491.

[25] B. Teissier, Sur la classification des singularités des espaces analytiques complexes. Proceedings of the international congress of mathematicians, August 16-24, 1983, Warszawa.

[26] B. Teissier, Résolution simultanée 1 et 2, Lecture Notes in Mathematics 777, Springer-Verlag 1980, pp. 71-146.

[27] B. Teissier, Cycles évanescents, sections planes, et conditions de Whitney. Singularités à Cargèse 1972, Astérisque 7-8, S. M. F. Paris.

[28] B. Teissier, The hunting of invariants in the geometry of discriminants, Real and Complex singularities, Proceedings of the Nordic Summer School, P. Holm, Oslo 1976, pp. 565-677.

[29] G. N. Tyurina, Absolute isolatedness of rational singularities and triple rational points, Func. Anal. Appl. 2 (1968), 324-332.

[30] H. Whitney, Tangents to an analytic Variety, Ann. of Math. 81 (1965), 496-549.

[31] S. S.-T. Yau, On maximally elliptic singularities, Trans. of the Amer. Math. Soci. Vol. 257 2 (1980), 269-329.

[32] O. Zariski, P. Samuel, Commutative algebra, Vol. 2, D. Van Nostrand, Princeton 1960.

\author{
Jawad Snoussi \\ CMAF, Universidade de Lisboa \\ Av. Prof. Gama Pinto 2 \\ 1649-003 Lisboa \\ Portugal \\ e-mail: jsnoussi@lmc.fc.ul.pt
}

(Received: February 21, 2000) 